\title{
Funktionale Klassifizierung von Kleinstädten in Deutschland. Ein methodischer Vergleich
}

\section{Functional classification of small towns in Germany. A methodological comparison}

https://doi.org/10.2478/rara-2020-0032

Eingegangen: 24. April 2020, Angenommen: 13. Oktober 2020

\begin{abstract}
Kurzfassung: Die Entwicklung von deutschen Kleinstädten hinsichtlich ihrer ökonomischen, demographischen und sozialen Ausstattung ist ein bislang eher unbeachteter Themenbereich. Es werden oftmals Kleinstädte in ländlichen und peripheren Lagen thematisiert, weil man innen ausschließlich hier eine gewisse Rolle zuweist. Kleinstädte in zentralen Lagen hingegen werden in der Forschung unter dem suburbanen Raum subsumiert und damit selten hinsichtlich ihrer spezifischen Funktionen betrachtet. In diesem Beitrag werden alle Kleinstädte in Deutschland hinsichtlich wichtiger Funktionen klassifiziert. Die Typisierung erfolgt mittels einer Clusteranalyse, die auf zentralen Faktoren basiert, welche sich auf ein breites Indikatorenset stützen. Bezugspunkte sind dabei das klassische Verfahren der Hauptkomponentenanalyse sowie als Alternativmethode die geographisch gewichtete Hauptkomponentenanalyse. Im methodischen Vergleich zeigt sich, dass die Alternative - auch aufgrund des hohen Rechenaufwands - noch keine geeignete Methode ist, um räumliche Zusammenhänge zwischen den Variablen abzubilden und die Kleinstädte hinsichtlich bestehender funktionaler Ausstattungen in ihrem Umland besser beschreiben zu können. Hingegen erzeugt die in der Literatur vorzufindende Vorgehensweise aus Hauptkomponentenanalyse, Clusteranalyse und Diskriminanzanalyse gut abgrenzbare und erklärbare Lösungen zur Bildung von Kleinstadttypen in Deutschland.
\end{abstract}

Schlüsselwörter: Kleinstadt, funktionale Klassifizierung, geographisch gewichtete Hauptkomponentenanalyse, Hauptkomponentenanalyse, Clusteranalyse

\begin{abstract}
The development of small towns in Germany in terms of their economic, demographic and social endowment is a subject area that has been rather neglected so far. What is more, research often focuses exclusively on small towns in rural and peripheral regions, because this is the only place where they are assigned a certain role. Small towns in central areas, on the other hand, are subsumed under suburbia in research and are thus rarely considered in terms of their specific functions. In this paper, all small towns in Germany are classified with regard to important functions. The typification is done by means of a cluster analysis based on central factors, which in turn are tied to a broad set of indicators. Reference points are the classical method of principal component analysis and, as an alternative method, to geographically weighted principal component analysis. The methodological comparison shows that the alternative method - also due to the high computational effort - is not yet a suitable method for
\end{abstract}

\footnotetext{
*Corresponding author: Dr. Philipp Gareis, Justus-Liebig-Universität Gießen, Institut für Geographie, Bereich Raumplanung und Stadtgeographie, Senckenbergstraße 1, 35390 Gießen, Deutschland, E-Mail: philipp.gareis@geogr.uni-giessen.de, ORCID: 0000-0001-5093-992X

Antonia Milbert, Bundesinstitut für Bau-, Stadt- und Raumforschung, Deichmanns Aue 31-37, 53179 Bonn, Deutschland
} 
describing spatial relationships between the variables and for small towns with regard to existing functions in their surrounding areas. On the other hand, the approach of the principal component analysis, cluster analysis and discriminant analysis, which is found in the literature, produces well defined and explainable solutions for the formation of different types of small towns in Germany.

Keywords: Small town, Functional classification, Geographically weighted principal component analysis, Principal component analysis, Cluster analysis

\section{Einleitung}

Untersuchungen zu Kleinstädten spielen bislang eine eher untergeordnete Rolle in der nationalen und internationalen Forschung (Bell/Jayne 2009: 683; Steinführer/Porsche/Sondermann 2019: 3). Vorherrschend ist dabei eine Dichotomie: einerseits eine Stadtforschung, die sich auf Großstädte fokussiert, und andererseits eine Forschung zum ländlichen Raum. Die „systematische Aufmerksamkeitslücke" (Porsche/Milbert/Steinführer 2019: 15) zu Kleinstädten ist allerdings nicht nur eine Frage mangelnder Forschung, sondern vielmehr einer fehlenden Rezeption bestehender Literatur zu Kleinstädten (vgl. ausführlich Herrenknecht/Wohlfahrt 2005: 7).

Ohne dass die Forschung also die Rolle und besonderen Funktionen der Kleinstädte empirisch belegen kann, wird Kleinstädten per se eine hohe Bedeutung beigemessen: In Zeiten steigender Immobilienpreise in Groß- und Mittelstädten sowie im Einzugsbereich von Metropolen und Regiopolen sollen in innen vor allem ausreichend Wohnungen bereitgestellt werden und Platz für Betriebe bieten, für die in den großen Zentren keine Flächen (mehr) existieren oder die dort zu teuer sind (Göddecke-Stellmann 2011: 5; Fehlberg/Mießner 2015: 26). Zugleich sollen sie eine lebenswerte und funktionale Umgebung schaffen und eine attraktive naturräumliche Ausstattung sichern (Porsche/Milbert 2018: 6). In peripheren Regionen sollen sie zentrale Ankerpunkte der wirtschaftlichen und gesellschaftlichen Entwicklung bilden (Empirica 2017: 7; Porsche/Milbert 2018: 6). Zusammengefasst wird von Kleinstädten erwartet, städtische Funktionen und Ambiente zu bieten und dabei dörfliche oder ländliche Rückzugsräume zu sein (Wirth/ Elis/Müller et al. 2016: 62-63).

„Kleinstädte sind kein homogener Stadttyp - weder hinsichtlich ihrer Größe, ihrer Geschichte und ihrer Stadtstruktur noch bezogen auf ihre aktuelle Entwicklung" (Porsche/Milbert/Steinführer 2019: 11). Aring (2003: 272) stellte hierzu fest, dass die Einwohnerzahl allein kein Kriterium einer Zentren- oder Knotenfunktion sein kann. Ein Zentrum kann weder ausschließlich nach der
Bevölkerungszahl noch ausschließlich innerhalb administrativer Grenzen gefasst werden; bei der Beurteilung müssen auch die Siedlungsstrukturen, die sozialräumlichen Systeme und das Umfeld, die ein Stadtzentrum mit einem Hinterland zu einer Kleinstadt formen können, einbezogen und hiernach unterschieden werden (Servillo/Atkinson/Smith et al. 2014: 3-4). Kleinstädte in zentralen Lagen werden stark durch die unterschiedlichen Entwicklungsdynamiken ihrer nahegelegenen Großund Mittelstädte beeinflusst (Schmitz-Veltin 2015: 82). Einerseits können sie von den Reurbanisierungsprozessen der vergangenen Jahre mit der Konzentration der demographischen und wirtschaftlichen Entwicklung auf Groß- und Mittelstädte, und hier wiederum insbesondere auf größere Agglomerationen und Metropolregionen, profitieren (Porsche/Milbert 2018: 14). Andererseits können sie Funktionen von Großstädten ,ausleihen", indem sie Märkte, Wissen und Netzwerke der nahegelegenen Großstädte oder Metropolen nutzen, über die sie selbst nicht verfügen. Dies wurde auch in Deutschland im Rahmen der Borrowing-Size-Debatte nachgewiesen (Volgmann/Rusche 2020: 76). Kleinstädte außerhalb der Agglomerationen stehen dagegen häufiger im Sog des demographischen Wandels (Reichert-Schick 2010: 156; Harfst/Wirth 2014: 466; Leibert 2019: 20), jedoch ergeben sich auch hier, bedingt durch verschiedene demographische Ausgangslagen und unterschiedliche Wanderungsgewinne oder -verluste, höchst unterschiedliche Entwicklungsverläufe (Winkler-Kühlken/ Diller/Gareis et al. 2019: 22). Kleinstädte in peripheren Regionen besitzen oftmals wichtige Funktionen für ihr Umland und versorgen dieses mit, trotz teilweise abnehmender Funktionsumfänge (Kühn/Milstrey 2015: 187; Wirth/Elis/Müller et al. 2016: 62). Aufgrund dieses Spannungsfeldes werden Kleinstädte auch als "chronischer Patient der Regionalentwicklung" (Wirth/Elis/Müller et al. 2016: 63) bezeichnet, über den es weitere großräumige Erkenntnisse zu gewinnen gilt. Diese sehr unterschiedlichen Ausgangssituationen führen zu folgenden zentralen Fragestellungen: 
- Welche Funktionen besitzen Kleinstädte und wie verteilen sich die Kleinstädte in Deutschland?

- Können neue methodische Ansätze einen Mehrwert an Erkenntnissen bezüglich der Einordnung der Kleinstädte in Deutschland liefern?

Der Ad-hoc-Arbeitskreis „Kleinstadtforschung“ der Akademie für Regionalentwicklung in der LeibnizGemeinschaft (ARL) empfiehlt, dass Kleinstädte „typisiert werden, um sie vergleichend und abstrahierend zu untersuchen“ (Porsche/Steinführer/Dehne et al. 2019: 52). Diese Empfehlung aufgreifend wird in diesem Beitrag ein methodischer Ansatz zur Klassifikation der Kleinstädte in Deutschland anhand eines umfassenden Indikatorensatzes präsentiert, der neben Bau-, Siedlungs-, Bevölkerungs- und Sozialstrukturindikatoren auch Indikatoren zu Erreichbarkeiten, Wirtschaft, Arbeitsmarkt, Infrastrukturausstattung und Versorgungsstruktur enthält. Mit diesem Ansatz werden alle 2.106 Kleinstädte in Deutschland typisiert. Da für eine Klassifikation von Objekten die Kriterienwahl maßgeblich ist, liegt hier der Fokus auf der Bestimmung der Faktoren. Dabei werden neben der klassischen, globalen Hauptkomponentenanalyse (PCA) auch verschiedene geographisch gewichtete Hauptkomponentenanalysen (GWPCA) getestet. Um den Ansatz des Borrowing Size bzw. des Einflusses der Nachbarschaft berücksichtigen zu können, werden die Hauptkomponenten für alle rund 4.500 Einheitsgemeinden und Gemeindeverbände Deutschlands ermittelt. Von der GWPCA wird erhofft, dass bei der Bestimmung der Hauptkomponenten das Umland direkt in diesem Maß berücksichtigt werden kann. Auf der Basis der so extrahierten Hauptkomponenten werden anschließend nur die Kleinstädte über eine Clusteranalyse gruppiert und diese Gruppen mittels Diskriminanzanalyse überprüft. Dieses Vorgehen ist neuartig, denn speziell zur GWPCA gibt es bisher nur wenige Vergleichsanalysen.

Zunächst wird in Kapitel 2 die Problemstellung schärfer umrissen und darauf aufbauend folgt in Kapitel 3 die Beschreibung der Daten und des methodischen Vorgehens. In Kapitel 4 werden die Ergebnisse der multivariaten Analysen vorgestellt und die räumliche Verortung interpretiert. Abschließend werden in Kapitel 5 ein Fazit gezogen, die Vor- und Nachteile der vorgestellten Klassifikationen dargelegt, ein Forschungsausblick sowie anwendungsbezogene Handlungsempfehlungen zur Kleinstadtklassifikation formuliert.

\section{Problemstellung}

Der Bedarf an einer Gesamtklassifikation von Städten über die funktionale Einordnung einzelner Städte hinaus wurde bereits Mitte des letzten Jahrhunderts erkannt. Mit der ersten Klassifikation von Städten über ein Set mit verschiedenen Statistiken aus dem Bevölkerungszensus und Unternehmensbefragungen markierte die Analyse von Harris (1943) den Beginn der multikriteriellen Distinktionen von Städten. In seiner Untersuchung bediente er sich noch der Setzung von Mindeststandards für die Bestimmung verschiedener funktionaler Stadttypen (Harris 1943: 86). Frühe Klassifizierungen beschränkten sich auf ökonomische Faktoren und auf den Arbeitsmarkt, was auch lange der besseren Verfügbarkeit der Wirtschafts-, Berufs- und Arbeitsmarktstatistiken geschuldet war (Smith 1965: 546-547). Denn Hauptziel der Typisierungen war lange, die spezifische Rolle der Städte innerhalb eines Städtesystems bezüglich der territorialen Arbeitsteilung zu identifizieren (Schulze/ Margraf 2000: 43). Diese fordistische Sichtweise wich nach und nach einer stärkeren Fokussierung auf spezifische Funktionen, die vor allem auch Klein- und Mittelstädten eine neue Bedeutung als Standorte wirtschaftlicher Entwicklung zuschreiben (Maillat 1998: 127; Meili/Mayer 2017: 317). Seit den 1990er-Jahren steigt die Anzahl an Literatur, in der Entwicklungschancen von Städten aufgrund ihrer sozialen Komponenten beispielsweise als Wohn- und Lebensstandorte thematisiert (z. B. Schulze/Margraf 2000; Gatzweiler/Adam/Milbert et al. 2012; Adam/Berndgen-Kaiser/Jochimsen et al. 2018) oder auf demographische Entwicklungen und Strukturen fokussiert werden (Blotevogel 2006; Schultz/Brandt 2017). Erwähnt seien auch die Ansätze, die Städte aufgrund ihrer spezifischen Lage (z. B. Grenz- oder Alpenlage) typisieren (Messerli 1999).

Neben diesen thematisch spezifischen Ansätzen werden immer wieder multikriterielle und mehrdimensionale Klassifikationen erarbeitet (z. B. Moser/Scott 1961; Geyler/Warner/Brandl et al. 2008; Schultz/Brandt 2017). Die thematisch wohl umfangreichste Klassifikation, der "Wegweiser Kommune" der Bertelsmann-Stiftung, nutzt neun Oberkategorien: Bildung, Demographischer Wandel, Finanzen, Integration, Nachhaltigkeit, Pflege, Soziale Lage, Wirtschaft und Arbeit. ${ }^{1}$

Um die große Zahl der Indikatoren zu strukturieren, stellten zuerst Moser und Scott (1961: 84-88) und nachfolgend andere Autoren (Andrews 1971: 272; Bähr 1971: 250; Schwippe 1979: 122-124; Grove/Roberts 1980:

1 https://www.wegweiser-kommune.de/ (12.09.2020). 
77-78; Blotevogel 2006: 20) der Clusteranalyse eine Hauptkomponentenanalyse (PCA) voran. Allerdings kritisierte bereits Smith (1965: 547) die Nichtberücksichtigung der geographischen Lage der Städte zueinander sowie die Wichtigkeit des Hinterlands. So stellten Geyler, Warner, Brandl et al. (2008: 51) in ihrer Analyse mittels PCA und Clusteranalyse Unschärfen bei der Klassifizierung fest, insbesondere bei Kleinstädten im suburbanen Raum, die mit den gewählten Verfahren nicht erklärt werden konnten.

Ansätze, die die funktionale Ausgestaltung des Umlandes (bzw. der gesamten Stadtregionen) in die Untersuchung miteinbeziehen, sind mehrheitlich auf sehr lokale Untersuchungsräume begrenzt und betrachten zudem eher große Metropolregionen und weniger Kleinstädte (Volgmann 2014: 31-34; Jiang/Alves/Rodrigues et al. 2015: 38). Arbeiten, die hingegen explizit Kleinstädte in ihrem regionalen Kontext betrachten, sind fast alle als Fallstudien konzipiert (z. B. Brombach/ Jessen 2005: 482). Meili und Mayer (2017: 320) lösen das Problem der regionalen Bezüge über eine Clusterung von Kleinstädten mit anschließender Varianzanalyse hinsichtlich unterschiedlicher Indikatoren, die die Austauschbeziehungen zwischen Städten abbilden. Winkler-Kühlken, Diller, Gareis et al. (2019: 19-20) beziehen erstmals sowohl die großräumige Lage als auch die Wirtschaftsstruktur der Regionen in die Klassifikation der Kleinstädte mit ein. Aus der Kombination von Lage, Regionstyp und Kleinstadttyp ergeben sich jedoch sehr viele, teils singulär besetzte Kleinstadttypen (WinklerKühlken/Diller/Gareis et al. 2019: 34), weshalb dieses Vorgehen als nicht ausbaufähig erachtet wird.

Es besteht also ein hoher Bedarf dahingehend, die große heterogene Gruppe der Kleinstädte weiter zu differenzieren und daraus ihre spezifischen Funktionspotenziale abzuleiten. Obwohl sich eine PCA zur Strukturierung und Verdichtung eines mehrdimensionalen und multikriteriellen Indikatorensatzes mit anschließender Clusteranalyse als gängiges Verfahren etabliert hat, muss die fehlende Berücksichtigung des regionalen Kontextes in vielen Analysen als Mangel betrachtet werden. Wie oben dargelegt, ist es für die Entwicklung von Kleinstädten bedeutsam, mit welchen Nachbarn sie konkurrieren, ob sie sich übergeordneter Netzwerke und Ausstattungen bedienen können (Volgmann/Rusche 2020: 61) oder ob sie das Zentrum einer dünn besiedelten, strukturschwachen Region darstellen. Ein Verfahren, die Ausstattung der Nachbargemeinden mit einzubeziehen, ist die GWPCA.

\section{Methodik}

Im Folgenden wird das methodische Vorgehen des Klassifizierungsansatzes beschrieben. Da auf der Ebene der Kleinstädte noch keine Erfahrungen mit der GWPCA vorliegen, wird zum Vergleich die für Gemeinde- und Städteklassifikationen vielfach erprobte PCA durchgeführt. Dadurch können die Ergebnisse der GWPCA besser beurteilt werden.

\subsection{Daten}

Angelehnt an bestehende multikriterielle Konzepte (Moser/Scott 1961: 84-88; Blotevogel 2006: 19-29; Gatzweiler/Adam/Milbert et al. 2012: 39-41; Winkler-Kühlken/ Diller/Gareis et al. 2019: 26-27) werden dort verwendete Indikatoren aus folgenden Bereichen für die Kleinstadttypik in Betracht gezogen: Bau- und Siedlungsstruktur, Bevölkerungs- und Sozialstruktur, Erreichbarkeiten/ Lage, Wirtschaft und Arbeitsmarkt, Verflechtungen über Ein- und Auspendlerbeziehungen sowie Infrastrukturausstattung der Grundversorgung und der mittleren und höherwertigen Versorgung. Die Auswertung erfolgt auf der Ebene der Einheitsgemeinden und Gemeindeverbände, um die Zuordnung des Bundesinstituts für Bau-, Stadt- und Raumforschung (BBSR) zum Stadt- und Gemeindetyp verwenden zu können. Das BBSR entwickelte diese Abgrenzung als statistische Vergleichskategorie in seiner Funktion als für die Raumbeobachtung des Bundes zuständige Behörde (Steinführer/Porsche/ Sondermann 2019: 6), sodass sie in der empirischen Forschung weitgehend anerkannt ist. Kleinstädte sind Einheitsgemeinden und Gemeindeverbände mit im Kern 5.000 bis unter 20.000 Einwohnern oder mindestens einer mittelzentralen Teilfunktion. ${ }^{2}$ Diese Abgrenzung ermöglicht einerseits, einen in der Forschung anerkannten Zugang zu den Kleinstädten zu nutzen, und stellt andererseits sicher, dass die Daten für die bundesweite Betrachtung flächendeckend auf Gemeindeebene zur Verfügung stehen. Datengrundlage ist dabei die INKARDatenbank. ${ }^{3}$ Insgesamt können 44 der in der Literatur (siehe oben) vorgeschlagenen Indikatoren mit verfügbaren Daten abgebildet werden (vgl. Tabelle 1). Für die

2 Raumabgrenzungen des BBSR: Stadt- und Gemeindetyp; https://www.bbsr.bund.de/BBSR/DE/forschung/raumbeobachtung/ Raumabgrenzungen/deutschland/gemeinden/StadtGemeindetyp/ StadtGemeindetyp.html?nn=2544954 (15.09.2020).

3 INKAR = Indikatoren und Karten zur Raum- und Stadtentwicklung; https://www.inkar.de (12.09.2020). 
rechenaufwendige GWPCA ist es jedoch erforderlich, die Datenfülle zu begrenzen, sodass parallel ein auf 13 Indikatoren reduziertes Datenset verwendet wird. Diese Indikatoren sind in Tabelle 1 grau markiert.

Bei den Variablen im Bereich Infrastruktur/Versorgung handelt es sich mehrheitlich um eine Auszählung betreffender Einrichtungen, die auf die ansässige Bevölkerung bezogen werden. Nur bei einigen Einrichtungsarten ist eine Qualifikation über Größe (z. B. Studierende an Hochschulen, Schüler an Schulen, Sitzplätze in Kinos) oder Spezialisierung (z. B. Krankenhäuser, Bibliotheken) möglich. Insbesondere stärker spezialisierte Einrichtungen, die eher als oberzentrale Einrichtungen in Groß- und Mittelstädten zu vermuten sind, können Kleinstädten ein besonderes Potenzial bieten und werden deshalb als Kriterium aufgenommen.

Sowohl zwischen den 44 als auch zwischen den 13 Variablen bestehen untereinander Korrelationen, die auf eine Redundanz räumlicher Informationen und/oder das Vorhandensein latenter Faktoren schließen lassen. Im Folgenden wird das Vorgehen zur Informationsverdichtung erörtert.

\subsection{Globale Hauptkomponentenanalyse (PCA) und geographisch gewichtete Hauptkomponentenanalyse (GWPCA)}

Mithilfe der PCA sollen stark miteinander korrelierende Variablen zu Faktoren bzw. Hauptkomponenten zusammengefasst werden (Backhaus/Erichson/Plinke et al. 2016: 386). Die PCA ist eine Spezialform der explorativen Faktorenanalyse. Es werden neue Variablen - die Hauptkomponenten - als Linearkombinationen aus allen Variablen gebildet, die erstens auf unterschiedlichen Indikatoren besonders hoch laden, und zweitens weitestgehend nicht miteinander korreliert sind. Sie beschreiben damit, wie die Kleinstädte mit den extrahierten Komponenten ausgestattet sind (vgl. Wolff/Bacher 2010: 348). Die Verengung auf strukturgebende Komponenten unter Nutzung der Gesamtvarianz des Datenmaterials ist der Hauptgrund für den Einsatz einer PCA (Wolff/ Bacher 2010: 349). Für die Analyse werden die Indikatoren zunächst standardisiert. Durch die Standardisierung kann zur Berechnung der Zusammenhänge und Extraktion der Hauptkomponenten die Kovarianzmatrix genutzt werden. Dieses Varimax-Verfahren wurde zur Erzeugung der Einfachstruktur der Hauptkomponenten zur Abgrenzung orthogonaler und damit unkorrelierter und voneinander unabhängiger Hauptkomponenten eingesetzt.
Um das Umfeld einer Kleinstadt in der Klassifizierung berücksichtigen zu können, wird die GWPCA eingesetzt. Dieses von Fotheringham, Brunsdon und Charlton (2002) entwickelte und von Lloyd (2010) erstmals empirisch eingesetzte Verfahren ermöglicht es, die Indikatoren geographisch mithilfe von Entfernungsdistanzen zu gewichten und somit geographische Besonderheiten in den Datenmustern erkenntlich zu machen. Zugehörige Gleichungen und Unterschiede zur PCA können unter anderem bei Fotheringham, Brunsdon und Charlton (2002: 196-202), Lloyd (2010: 394) und Harris, Brunsdon und Charlton (2011: 1720) entnommen werden. Es werden über dieses Verfahren geographisch gewichtete Standardabweichungen, Kovarianzen und somit auch Korrelationskoeffizienten berechnet, um den Einfluss räumlich naher Einheiten für jeden einzelnen Standort zu ermitteln. Der Haupteffekt der GWPCA ist es, gegebene räumliche Autokorrelation einbeziehen zu können (Harris/Brunsdon/Charlton 2011: 1720). Es soll somit ein besseres Verständnis der untersuchten Einheiten unter Berücksichtigung nahe gelegener Untersuchungseinheiten gewährleistet werden (Harris/Clarke/ Juggis et al. 2015: 148). Dabei macht die GWPCA von Moving-window-Techniken Gebrauch, die es ermöglichen, dass alle im Umkreis einer bestimmten Bandbreite festgelegten Werte mit einer räumlichen Distanzmatrix oder Kernelfunktion gewichtet werden (Gollini/Lu/Charlton et al. 2015: 4-5). Bisher gibt es erst wenige Versuche, die GWPCA auf administrative Gebietseinheiten anzuwenden (Harris/Brunsdon/Charlton 2011: 1722; Li/Cheng/ Wu 2016: 235).

Das größte Problem besteht in der Wahl der räumlichen Bandbreite. Des Weiteren muss vorab die Zahl der Hauptkomponenten festgelegt werden. In Analogie zu Li, Cheng und Wu (2016: 241-242) und Tsutsumida, Harris und Comber (2017: 1067) wird hier die gleiche Zahl an Hauptkomponenten gewählt, wie sie auch bei der globalen PCA berechnet wurde. In diesem Fall erfolgte die Auswahl über den Scree-Plot, die Komponenten mit einem Eigenwert größer 1 und der erklärten Gesamtvarianz. Es wird hierzu das GWmodel-Paket der Software R genutzt, um die Bandbreite automatisch zu bestimmen (Gollini/Lu/Charlton et al. 2015: 5). Wie weiter unten gezeigt wird, ist das Gesamtdatenmaterial plus automatisch bestimmter Bandbreite wenig zielführend. Es erfolgt daher eine weitere alternative Messung über erstens einen reduzierten Datensatz mit 13 Indikatoren und zweitens eine manuelle Einstellung der Bandbreite von alternativ $10 \mathrm{~km}, 25 \mathrm{~km}$ sowie $50 \mathrm{~km}$. Diese Bandbreiten orientieren sich an den durchschnittlichen Pendeldistanzen, die gemäß Statistischem Bundesamt 
Tabelle 1: Liste der verwendeten Bereiche, Indikatoren und Variablen

\begin{tabular}{|c|c|c|c|}
\hline \multirow[t]{2}{*}{ Bereich } & \multicolumn{3}{|l|}{ Indikator } \\
\hline & Name & Bezeichnung & Maßeinheit \\
\hline \multirow{3}{*}{ 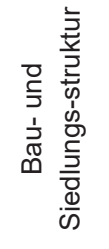 } & Mehrfamilienhäuser & $\begin{array}{l}\text { Anteil der Wohngebäude mit drei und mehr Wohnungen an allen } \\
\text { Wohngebäuden }\end{array}$ & $\%$ \\
\hline & $\begin{array}{l}\text { neu errichtete } \\
\text { Mehrfamilienhäuser }\end{array}$ & $\begin{array}{l}\text { Anteil fertiggestellter Wohngebäude mit drei und mehr Wohnungen an } \\
\text { allen fertiggestellten Wohngebäuden }\end{array}$ & $\%$ \\
\hline & Siedlungsdichte & Einwohner je Siedlungs- und Verkehrsfläche & Einwohner/km² \\
\hline \multirow{7}{*}{ 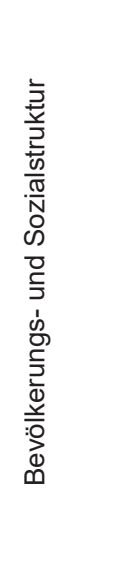 } & Beschäftigtenquote & $\begin{array}{l}\text { sozialversicherungspflichtig Beschäftigte am Wohnort je Einwohner im } \\
\text { erwerbsfähigen Alter }\end{array}$ & je 100 Einwohner \\
\hline & Langzeitarbeitslose & $\begin{array}{l}\text { Anteil der Arbeitslosen, die ein Jahr und länger arbeitslos sind, an allen } \\
\text { Arbeitslosen }\end{array}$ & $\%$ \\
\hline & $\begin{array}{l}\text { Haushalte mit niedrigem } \\
\text { Einkommen }\end{array}$ & $\begin{array}{l}\text { Anteil der Haushalte mit einem monatlichen Einkommen unter } 1.500 € \\
\text { an allen Haushalten }\end{array}$ & $\%$ \\
\hline & Geschlechterproportion & $\begin{array}{l}\text { Verhältnis weibliche zu männlichen Einwohnern im Alter von } 20 \text { bis } \\
\text { unter } 40 \text { Jahre }\end{array}$ & Verhältniszahl \\
\hline & Durchschnittsalter & Durchschnittsalter der Bevölkerung & Jahre \\
\hline & Attraktivität des Wohnorts & Verhältnis der Zuzüge zu den Fortzügen & Verhältniszahl \\
\hline & Gesamtwanderungssaldo & Gesamtwanderungssaldo je 1.000 Einwohner & je 1.000 Einwohner \\
\hline \multirow{6}{*}{ 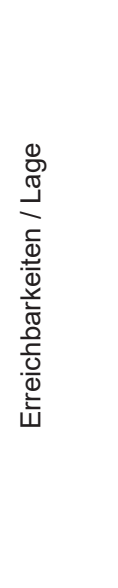 } & Bevölkerungspotenzial & $\begin{array}{l}\text { Verhältnis Bevölkerungspotenzial zum durchschnittlichen } \\
\text { Bevölkerungspotenzial des Bundes }\end{array}$ & Verhältniszahl \\
\hline & $\begin{array}{l}\text { Erreichbarkeit von } \\
\text { Autobahnen }\end{array}$ & Durchschnittliche Pkw-Fahrzeit zur nächsten Autobahnanschlussstelle & Minuten \\
\hline & $\begin{array}{l}\text { Erreichbarkeit von } \\
\text { Flughäfen }\end{array}$ & $\begin{array}{l}\text { Durchschnittliche Pkw-Fahrzeit zum nächsten internationalen Flughafen } \\
\text { in Deutschland }\end{array}$ & Minuten \\
\hline & $\begin{array}{l}\text { Erreichbarkeit von } \\
\text { Fernbahnhöfen }\end{array}$ & Durchschnittliche Pkw-Fahrzeit zum nächsten IC/ICE-Bahnhof & Minuten \\
\hline & $\begin{array}{l}\text { Erreichbarkeit von } \\
\text { Oberzentren }\end{array}$ & Durchschnittliche Pkw-Fahrzeit zum nächsten Oberzentrum & Minuten \\
\hline & $\begin{array}{l}\text { Erreichbarkeit von } \\
\text { Mittelzentren }\end{array}$ & Durchschnittliche Pkw-Fahrzeit zum nächsten Mittelzentrum & Minuten \\
\hline \multirow{8}{*}{ 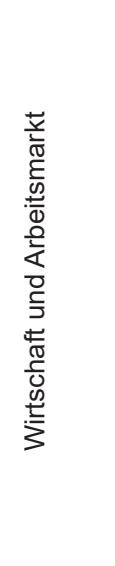 } & Arbeitslosigkeit & Arbeitslose je Einwohner im erwerbsfähigen Alter & je 1.000 Einwohner \\
\hline & Steuerkraft & Gemeindliche Steuerkraft je Einwohner & Euro je Einwohner \\
\hline & Gewerbesteuer & Gewerbesteuern je Einwohner & Euro je Einwohner \\
\hline & Arbeitsplatzzentralität & Verhältnis Einpendler zu Auspendler & Verhältniszahl \\
\hline & Pendlersaldo & Pendlersaldo je sozialversicherungspflichtig Beschäftigtem am Wohnort & je 100 Beschäftigte \\
\hline & Innergemeindliche Pendler & Anteil innergemeindlicher Pendler an allen Pendlern & $\%$ \\
\hline & Attraktivitätsradius & $\begin{array}{l}\text { durchschnittliche Distanz eines Einpendlers (Tagespendler unter } 150 \\
\text { km) }\end{array}$ & $\mathrm{Km}$ \\
\hline & Aktivitätsradius & $\begin{array}{l}\text { durchschnittlicher Arbeitsweg eines Auspendlers (Tagespendler unter } \\
150 \mathrm{~km} \text { ) }\end{array}$ & $\mathrm{Km}$ \\
\hline
\end{tabular}


Tabelle 1: Liste der verwendeten Bereiche, Indikatoren und Variablen

\begin{tabular}{|c|c|c|c|}
\hline \multirow[t]{2}{*}{ Bereich } & \multicolumn{3}{|l|}{ Indikator } \\
\hline & Name & Bezeichnung & Maßeinheit \\
\hline \multirow{20}{*}{ 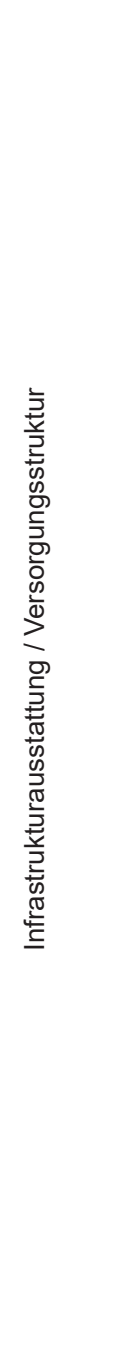 } & Polizeidienststellen & Anzahl der Polizeidienststellen je Einwohner & je 1.000 Einwohner \\
\hline & Jobcenter & Anzahl der Jobcenter je Einwohner & je 1.000 Einwohner \\
\hline & Gerichte & Anzahl der Gerichte je Einwohner & je 1.000 Einwohner \\
\hline & $\begin{array}{l}\text { Abfahrten des öffentlichen } \\
\text { Verkehrs }\end{array}$ & $\begin{array}{l}\text { Anzahl der Abfahrten an Haltepunkten des öffentlichen Verkehrs je } \\
\text { Einwohner }\end{array}$ & je 1.000 Einwohner \\
\hline & Bahn-Abfahrten & Anzahl der Bahn-Abfahrten je Einwohner & je 1.000 Einwohner \\
\hline & Breitbandversorgung & $\begin{array}{l}\text { Anteil der Haushalte mit Breitbandversorgung mit } 50 \text { Mbit/s an allen } \\
\text { Haushalten }\end{array}$ & $\%$ \\
\hline & $\begin{array}{l}\text { Krankenhäuser der } \\
\text { Grundversorgung }\end{array}$ & Anzahl der Krankenhäuser der Grundversorgung je Einwohner & je 1.000 Einwohner \\
\hline & $\begin{array}{l}\text { Krankenhäuser } \\
\text { Spezialversorgung }\end{array}$ & $\begin{array}{l}\text { Anzahl der Krankenhäuser mit mindestens Versorgungsstufe } 4 \text { (Voll- } \\
\text { und Spezialversorgung) je Einwohner }\end{array}$ & je 1.000 Einwohner \\
\hline & Apotheken & Anzahl der Apotheken je Einwohner & je 1.000 Einwohner \\
\hline & Bibliotheken & Anzahl der Bibliotheken je Einwohner & je 1.000 Einwohner \\
\hline & Spezialbibliotheken & Anzahl der wissenschaftlichen und Spezialbibliotheken je Einwohner & je 1.000 Einwohner \\
\hline & Hochschulen & Studierende an Universitäten und Hochschulen je Einwohner & je 1.000 Einwohner \\
\hline & Fachhochschulen & Studierende an Fachhochschulen je Einwohner & je 1.000 Einwohner \\
\hline & Grundschulen & Schüler in Grundschulen je Einwohner & je 1.000 Einwohner \\
\hline & weiterführende Schulen & Schüler in weiterführenden Schulen je Einwohner & je 1.000 Einwohner \\
\hline & berufsbildende Schulen & Schüler in berufsbildenden Schulen je Einwohner & je 1.000 Einwohner \\
\hline & Kinos & Anzahl der Kinos je Einwohner & je 1.000 Einwohner \\
\hline & Kinositzplätze & Anzahl der Kinositzplätze je Einwohner & je 1.000 Einwohner \\
\hline & Supermärkte & Anzahl der Supermärkte und Discounter je Einwohner & je 1.000 Einwohner \\
\hline & Shopping Center & $\begin{array}{l}\text { Anzahl der im Shopping Center Report geführten Shopping Center je } \\
\text { Einwohner }\end{array}$ & je 1.000 Einwohner \\
\hline
\end{tabular}

grau markiert: reduziertes Set mit 13 Indikatoren Quelle: Eigene Zusammenstellung nach INKAR

für $90 \%$ der Einwohnerinnen und Einwohner Deutschlands tägliche Aktionsradien widerspiegeln. ${ }^{4}$

Die beiden Hauptkomponentenanalysen werden nicht ausschließlich für die 2.106 Kleinstädte, sondern für alle 4.508 Einheitsgemeinden und Gemeindeverbände durchgeführt. Dies hat drei Gründe: Die Funktionen und Kennzahlen sind erstens so ubiquitär gewählt, dass sie auch als Distinktionskriterien für alle anderen Stadttypen genutzt werden können. Zweitens erstreckt sich der
Werteraum im Ergebnis über alle Stadt- und Gemeindegrößen; die Werte der Kleinstädte sind somit relativ zu inrer Rolle und Größe im Raum zu sehen. Kleinstädte sind drittens nicht losgelöst zu betrachten, sondern eingebettet im Raum. Dies trifft nicht nur, aber in besonderem Maße, für die Interpretation der Ergebnisse der GWPCA zu, kann aber auch für die PCA entsprechend gedeutet werden. 


\subsection{Clusteranalyse}

Für die Clusterung wird nur noch auf die Kleinstädte gemäß der Klassifikation des BBSR zurückgegriffen. Das Ziel der Clusteranalyse ist es, die sehr heterogene Gruppe der Kleinstädte in möglichst homogene Untergruppen aufzuteilen. Es stehen viele verschiedene Verfahren der Clusteranalyse zur Verfügung, von denen hier ein hierarchisch agglomeratives Verfahren gewählt wird. Hierzu wird der Ward-Algorithmus genutzt, welcher jede Fusion auf der niedrigsten Steigerung der Fehlerquadratsumme berechnet und so möglichst homogene Untergruppen garantiert. Das Ward-Verfahren wird wegen seiner konservativen Eigenschaften, der Erkennung der „wahren“ Gruppenzugehörigkeiten (Backhaus/ Erichson/Plinke et al. 2016: 298) und der Bildung von relativ gleich großen Gruppen häufig verwendet. Es ist zudem in der Lage, auch Ausreißer einer Gruppe zuzuordnen, weshalb diese vorher nicht unbedingt aus der Grundgesamtheit eliminiert werden müssen. Ausreißer können dabei vorab mit dem Single-Linkage-Verfahren identifiziert werden (Backhaus/Erichson/Plinke et al. 2016: 300). Im Datensatz befinden sich unter den Kleinstädten nur zwei Städte, die als Ausreißer gewertet, aber im Ward-Verfahren passenden Gruppen zugeordnet werden können. Wie von Backhaus, Erichson, Plinke et al. (2016: 298) empfohlen, wird als Distanzmaß für das Ward-Verfahren die quadrierte euklidische Distanz genutzt. Die Clusteranalyse wird vergleichend mit den Hauptkomponenten der PCA als auch mit den Hauptkomponenten aus der GWPCA gerechnet. Die Entscheidung zur optimalen Clusteranzahl stützt sich auf die Gegenüberstellung von 30 Cluster-Validitätsindizes, wie sie im R-Paket NbClust (Charrad/Ghazzali/Boiteau et al. 2014: 5-17) implementiert sind, sowie auf einer inhaltlichen Begutachtung alternativer Clusterlösungen.

Ein Nachteil der hierarchischen Clusterverfahren ist, dass einmal gebildete Fusionen von Einheiten im Verfahren nicht mehr gelöst werden. Eine Umsortierung zur Verkleinerung der Streuung innerhalb der Cluster wie bei partitionierenden Verfahren ist somit nicht möglich. Deshalb wird im Anschluss der hierarchischen Clusteranalyse sowohl ein kmeans-Clusterverfahren mit den Clustermittelwerten aus der Ward-Analyse als Startpartition (Backhaus/Erichson/Plinke et al. 2016: 284) als auch eine Umgruppierung der Kleinstädte über eine Diskriminanzanalyse (Backhaus/Erichson/Plinke et al. 2016: 95) mit den hier verwendeten Hauptkomponenten geprüft. Die Diskriminanzanalyse ermittelt die diskriminierenden Funktionen zur bestmöglichen Trennung bekannter Gruppen (Backhaus/Erichson/Plinke et al. 2016: 96). Die beiden Methoden ergänzen sich insofern, als mithilfe der Diskriminanzanalyse die von einer vorgeschalteten Clusteranalyse erzeugten Gruppen untersucht und die Gruppenzugehörigkeit der Objekte validiert werden kann: „Falsch“ gruppierte Kleinstädte können somit nachträglich in eine andere Gruppe eingeordnet werden (Backhaus/Erichson/Plinke et al. 206: 115). Die Prüfung und Optimierung einer gefundenen Clusterung mittels einer Diskriminanzanalyse setzten unter anderem Schneider und Scheibler (1983: 213) sowie BBSR (2016: 17) ein.

Zusammenfassend werden folgende sechs Hauptkomponentenanalysen durchgeführt:

- PCA mit 44 Indikatoren

- GWPCA mit 44 Indikatoren und automatisch bestimmter Bandbreite

- PCA mit 13 Indikatoren

- drei GWPCA mit 13 Indikatoren mit je einer Bandbreite von $10 \mathrm{~km}, 25 \mathrm{~km}$ und $50 \mathrm{~km}$

Nach Interpretation der Ergebnisse werden drei Clusteranalysen durchgeführt: auf Basis der PCA mit 44 Indikatoren, auf der Basis der PCA mit 13 Indikatoren und auf der Basis der GWPCA mit 13 Indikatoren und einer Bandbreite von $25 \mathrm{~km}$. Jede dieser Clusteranalysen wird über eine Diskriminanzanalyse korrigiert.

\section{Ergebnisse}

Nach der Erläuterung des Analysedesigns sollen zunächst die Ergebnisse der PCA mit denen der GWPCA vergleichend dargestellt werden. Die PCA auf der Basis der 44 Indikatoren ergibt nach Meinung der Autoren das plausibelste Ergebnis; die Hauptkomponenten und Cluster dieser Variante werden daher im Anschluss ausführlicher erläutert.

\subsection{Ergebnisse der PCA und GWPCA im Vergleich}

Die 44 Indikatoren lassen sich zu zehn Hauptkomponenten verdichten, die einen Eigenwert größer 1 (Kaiser-Kriterium) besitzen (vgl. Abbildung 1, linke Hälfte). Ein Eigenwert größer 1 bedeutet, dass die Hauptkomponente mehr zur Struktur beiträgt als eine einzelne Variable. Insgesamt erklären die zehn Hauptkomponenten knapp 60 \% der Gesamtvarianz der Variablen. Das Kaiser-Kriterium ist nur eines der möglichen Entscheidungshilfen für die Anzahl der zu bestimmenden Hauptkomponenten. Die Anzahl der Hauptkomponenten 

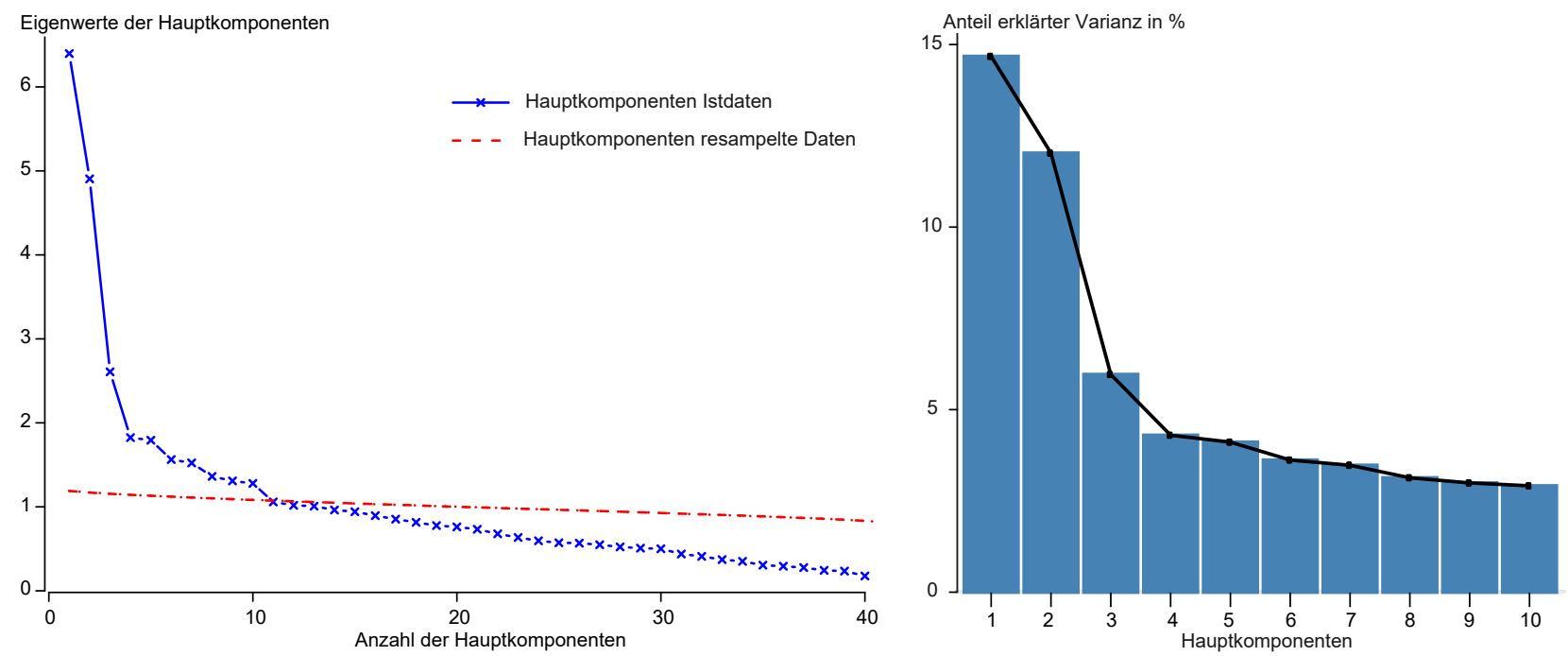

Abbildung 1: Scree-Plot der Eigenwerte (links) und der Varianz der zehn Hauptkomponenten (rechts) Quelle: Eigene Berechnungen nach INKAR

kann sich auch an der nach Reihenfolge abnehmenden Varianz orientieren (vgl. Abbildung 1, rechte Hälfte). Die größten Abstände in der erklärten Varianz finden sich zwischen der zweiten und dritten sowie zwischen der dritten und vierten Hauptkomponente. Mit den drei Hauptkomponenten würden aber nur $34 \%$ der Gesamtvarianz erklärt.

Meistens besteht die Schwierigkeit darin, den Hauptkomponenten eindeutige Namen auf Basis der am stärksten ladenden Indikatoren zu geben, was der Identifizierung der Gemeinsamkeiten bzw. Charakteristik dieser Hauptkomponente dient. Mehr oder weniger passende Bezeichnungen sind bei der globalen Hauptkomponente mit geringen Einschränkungen noch zu finden; die Hauptkomponenten der GWPCA sind dagegen deutlich schwieriger zu interpretieren. Da die Bezeichnungen treffend, aber umständlich sind, wird im weiteren Verlauf dennoch mit nummerierten Kürzeln der Hauptkomponenten operiert.

Eigenwerte und Interpretierbarkeit der Hauptkomponenten führen zur Entscheidung, bei der PCA auf Basis der 44 Indikatoren zehn Hauptkomponenten und bei der PCA mit 13 Indikatoren fünf Hauptkomponenten zu bestimmen. Die Anzahl der Hauptkomponenten der GWPCA wird denen der PCA angepasst, was nicht bedeutet, dass die Hauptkomponenten gleich strukturiert sind (Li/Cheng/Wu 2016: 241-242; Tsutsumida/ Harris/Comber 2017: 2). Die erste Hauptkomponente erklärt jeweils den größten Anteil an der Gesamtvarianz. Abbildung 2 illustriert die Verteilung der Score-Werte der jeweils ersten Hauptkomponente. Die erste Hauptkomponente aus dem vollen Indikatorensatz besitzt die stärksten Ladungen auf Indikatoren, die Dienstleistungen einer mittelzentralen Versorgung anzeigen, so die Zahl an Jobcenter, berufs- und weiterführende Schulen je Einwohner und die Nähe zum nächsten Mittelzentrum (linke Teilkarte). In den Großstädten sind andere Qualitäten an Einrichtungen vorhanden, sodass hier die Hauptkomponente niedrigere Werte aufweist als in kleineren Städten, genauso wie die meisten Landgemeinden. Klein- und Mittelstädte haben hier im Durchschnitt also die höchsten Faktorwerte.

Auf der Basis des reduzierten Indikatorensatzes laden Indikatoren der Dichte und Zentralität am stärksten auf der ersten Hauptkomponente (Anteil Mehrfamilienhäuser, Siedlungsdichte und Pendlersaldo, rechte Teilkarte). Man erkennt größere Gebiete stärkerer Dichte (Agglomerationen) genauso wie singulär dicht besiedelte Gemeinden und Städte im sonst weniger verdichteten Gebiet. Obwohl beide Teilkarten also unterschiedliche Aspekte der Städte anzeigen, erkennt man die unterschiedlichen regionalen Zusammenhänge sehr gut. Dies spricht für das Vorgehen, die Hauptkomponenten bundesweit auf der Basis aller Einheitsgemeinden und Gemeindeverbände zu bestimmen.

Ein allgemein anerkanntes Testverfahren zur Bewertung der Güte der GWPCA im Vergleich zur PCA liegt noch nicht vor (Demšar/Harris/Brunsdon et al. 2013: 118). Durch räumlich stark ausgeprägte Wirkungsmuster wirtschaftlicher, demographischer und sozialer Funktionen 
1. von 10 Hauptkomponenten aus 44 Indikatoren

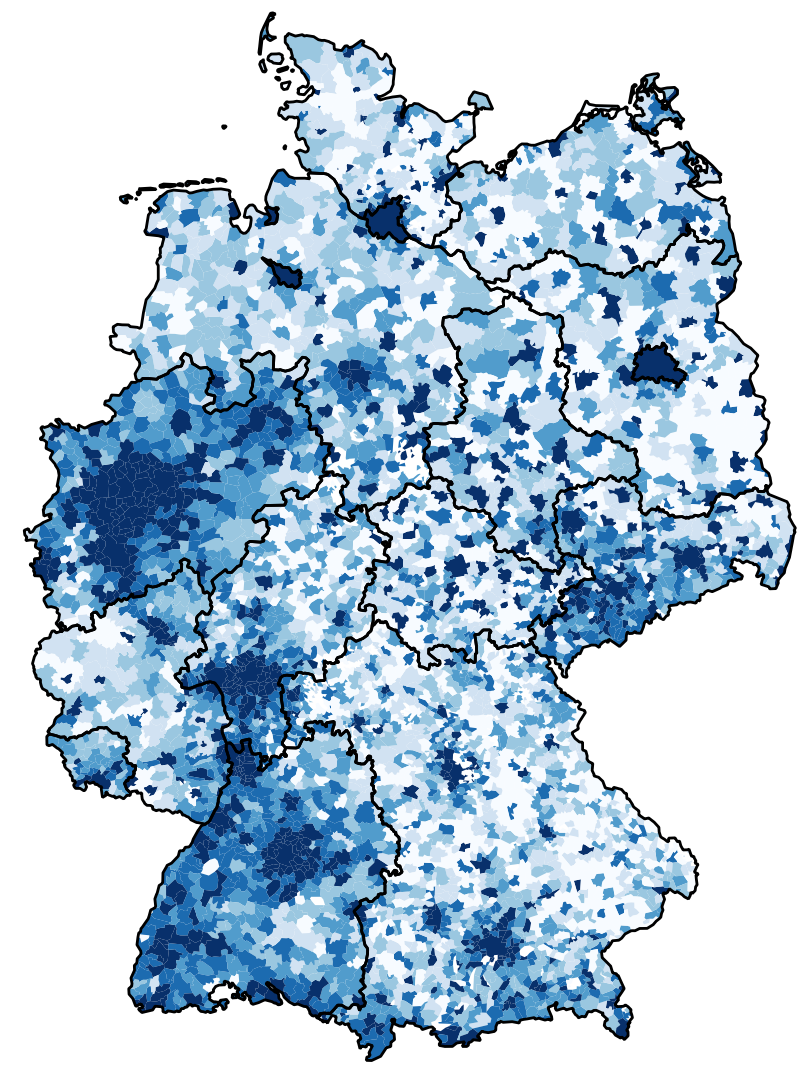

Score-Werte Hauptkomponente 1
$-4.954--0.722$
$-0.722--0.473$
$-0.473--0.240$
$-0.240-0.031$
$0.031-0.730$
$0.730-7.750$

1. von 5 Hauptkomponenten aus 13 Indikatoren

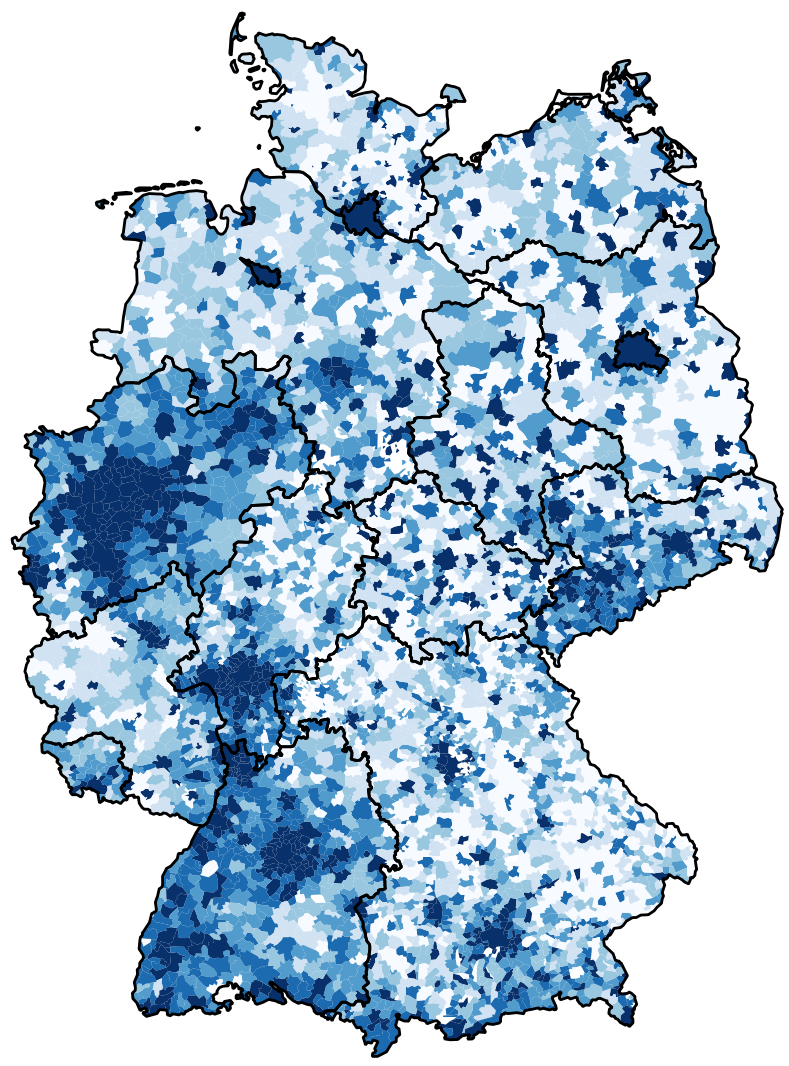

Score-Werte Hauptkomponente 1
$-5.274--0.855$
$-0.855--0.545$
$-0.545--0.205$
$-0.205-0.216$
$0.216-0.895$
$0.895-5.137$

Abbildung 2: Regionale Verteilung der ersten Hauptkomponente der globalen PCA auf der Basis von 44 Indikatoren (links) und auf der Basis von 13 Indikatoren (rechts)

Quelle: Eigene Berechnungen; Kartengrundlage: Bundesamt für Kartographie und Geodäsie ${ }^{5}$

in den verschiedenen Stadttypen in Deutschland ist von räumlichen Korrelationen zwischen den Komponenten auszugehen. Mit dem hohen Rechenaufwand für die GWPCA, welcher zum einen auf der Anzahl der Variablen, zum anderen auf der Anzahl der Untersuchungseinheiten (in diesem Fall Polygone) beruht, wurde die Reduktion der Variablen auf 13 Kernindikatoren begründet. Die Werte der Varianten für 10 und 25 Kilometer Bandbreite bei adaptivem Vorgehen gleichen sich den

5 https://gdz.bkg.bund.de/index.php/default/digitale-geodaten/ verwaltungsgebiete/verwaltungsgebiete-1-25-000-ebenen-stand31-12-vg25-ebenen.html (12.09.2020).
Berechnungen zufolge vollständig, für die Variante mit 50 Kilometer Bandbreite ergeben sich nur geringe Abweichungen. Für die weiteren Analyseschritte wird daher nur noch die Variante mit einer Bandbreite von 25 km genutzt.

Abbildung 3 zeigt die räumliche Verteilung der ScoreWerte der ersten Hauptkomponente der GWPCA auf der Basis des vollen Datensatzes und automatisch bestimmter Bandbreite (linke Teilkarte) sowie auf der Basis des reduzierten Indikatorensatzes und einer Bandbreite von $25 \mathrm{~km}$ (rechte Teilkarte). Die Karten zeigen ungewohnte räumliche Verteilungsmuster, die stärker an geographischen Gegebenheiten als an sozioökonomischen und 
1. von 10 Hauptkomponenten aus 44 Indikatoren

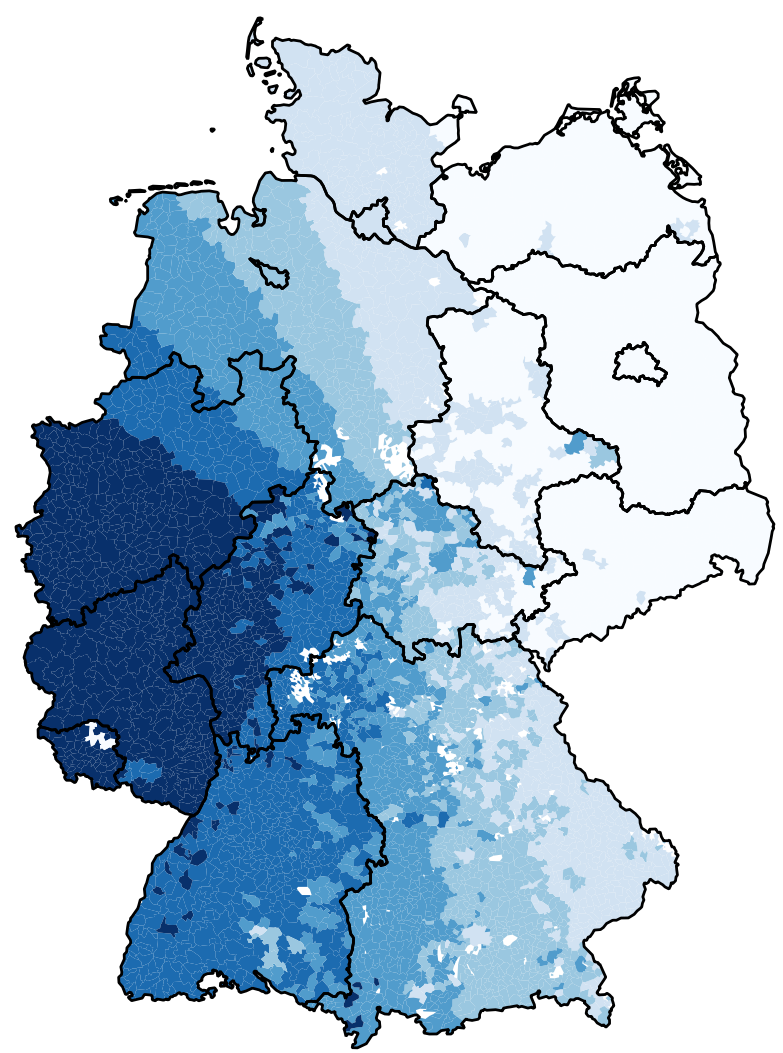

Score-Werte Hauptkomponente 1
$-0.302--0.299$
$-0.299--0.297$
$-0.297--0.296$
$-0.296--0.292$
$-0.292--0.281$
$-0.281--0.149$

1. von 5 Hauptkomponenten aus 13 Indikatoren

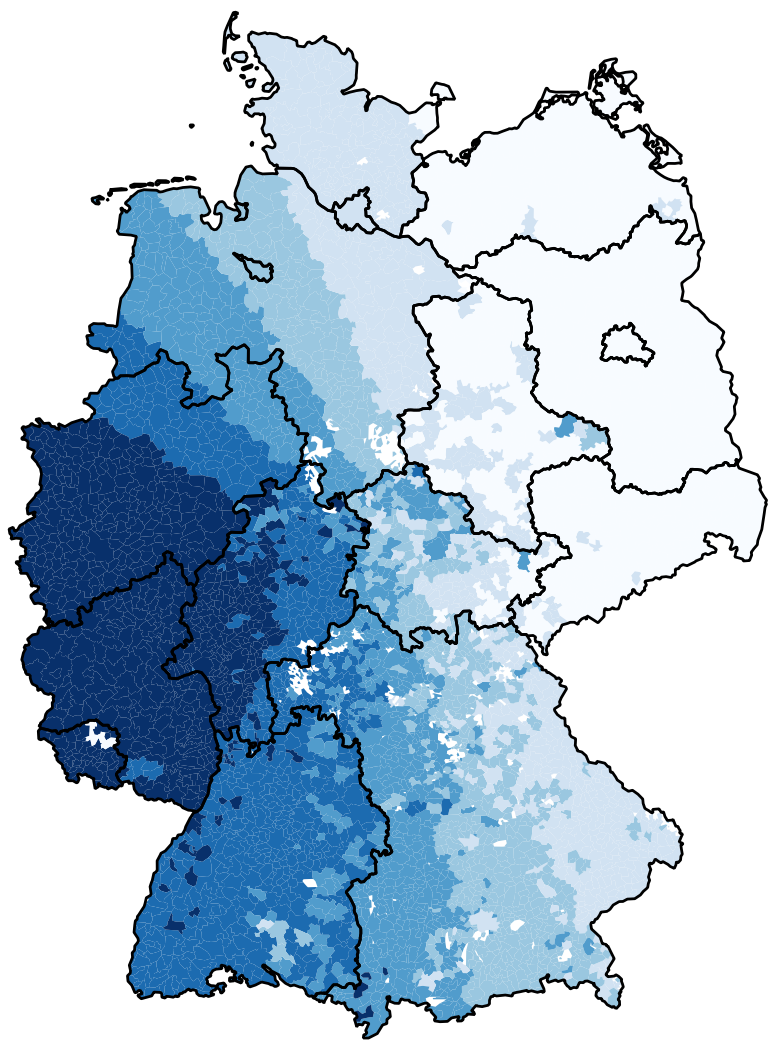

Score-Werte Hauptkomponente 1
$-0.472--0.470$
$-0.470--0.469$
$-0.469--0.468$
$-0.468--0.466$
$-0.466--0.465$
$-0.465--0.461$

Abbildung 3: Regionale Verteilung der ersten Hauptkomponente der GWPCA auf der Basis von 44 Indikatoren (links) und auf der Basis von 13 Indikatoren (rechts)

Quelle: Eigene Berechnungen; Kartengrundlage: Bundesamt für Kartographie und Geodäsie ${ }^{6}$

infrastrukturellen Werten ausgerichtet zu sein scheinen. Die Ladungen der Indikatoren sind deutlich niedriger als bei einer globalen PCA und nicht so eindeutig auf eine Hauptkomponente gerichtet. Die Hauptkomponente der linken Teilkarte lädt auf den Anteil innergemeindlicher Pendler, die Arbeitslosigkeit, die Arbeitsplatzzentralität, den Anteil der Mehrfamilienhäuser, das Pendlersaldo, die Zahl der Gerichte und berufsbildenden und weiterführenden Schulen. Könnte man hier eine gewisse Dichte und Zentralität herauslesen, so erstaunt, dass

6 https://gdz.bkg.bund.de/index.php/default/digitale-geodaten/ verwaltungsgebiete/verwaltungsgebiete-1-25-000-ebenen-stand31-12-vg25-ebenen.html (12.09.2020). alle Ladungen negativ sind und damit zum Teil der Zentralitätsvermutung widersprechen. Die Hauptkomponente der rechten Teilkarte lädt negativ auf den Indikatoren Arbeitslosigkeit, Mehrfamilienhäuser, Pendlersaldo und Anteil der Haushalte mit niedrigem Einkommen. Mit Ausnahme der Mehrfamilienhäuser sind gleichlaufend niedrige Werte dieser Hauptkomponente (negative Ladungen) in Ostdeutschland plausibel (ökonomisch schwächere Gemeinden), die geographische Häufung ähnlicher Werte in anderen Landesteilen in dieser Form dagegen weniger.

Allgemein lässt sich feststellen, dass die Hauptkomponentenladungen der GWPCA deutlich niedriger als bei der PCA sind. Sie schwanken mehr oder weniger zwi- 
schen $-0,6$ und 0,6. Deshalb kommt es häufig vor, dass ein Indikator ähnlich hoch auf mehrere Hauptkomponenten lädt.

Die automatisch ermittelte Bandbreite von $882 \mathrm{~km}$, welche 4.432 Wertepaare ergibt und somit beinahe der Grundgesamtheit entspricht, lässt darauf schließen, dass mit dem umfangreichen Datenmaterial dieses Vorgehen nicht zielführend ist (vgl. Tsutsumida/Harris/ Comber 2017: 1066). Aber auch die Berechnungen mit weniger Variablen und einer deutlich niedrigeren Bandbreite führen nicht zum erhofften Ergebnis. Beim weiteren Vorgehen erfolgt daher eine Konzentration auf die PCA und die Ergebnisse der GWPCA werden verworfen.

\subsection{Hauptkomponenten der globalen PCA}

Im Vergleich der beiden PCAs führt die Analyse mit 44 Indikatoren zu differenzierteren Hauptkomponenten als die auf der Basis von 13 Indikatoren. Die oberzentralen Einrichtungen, welche beim reduzierten Datensatz nicht berücksichtigt werden, laden alle auf der 8 . Hauptkomponente. Die Ladungen der einzelnen Indikatoren auf den Hauptkomponenten sind Tabelle 2 zu entnehmen.

Die finale Klassifikation wird daher mit den zehn Hauptkomponenten des umfassenden Datensatzes berechnet. Diese werden im Folgenden interpretiert: Hauptkomponente RC1 steht für die Ausstattung der Städte mit mittelzentralen Einrichtungen. Auf dieser Hauptkomponente laden die Zahl der Jobcenter, der Gerichte und der Schüler sowohl an weiterführenden als auch an berufsbildenden Schulen positiv. Negativ lädt der Indikator der Erreichbarkeit des nächsten Mittelzentrums. Dem Anteil der Binnenpendler wird weniger Bedeutung beigemessen, da dieser Indikator auch relativ stark auf dem Faktor RC8 lädt.

Hauptkomponente RC2 beschreibt die ZentrumPeripherie-Dimension. Auf dieser Hauptkomponente laden alle Erreichbarkeitsindikatoren zu höherwertigen Infrastrukturen (Autobahnen, Bahnhöfe, Flughäfen, Oberzentren). Negativ laden dagegen das Bevölkerungspotenzial und die Siedlungsdichte. Dass auch die Breitbandversorgung, das Geschlechterverhältnis und der Anteil an Mehrfamilienhäusern auf diesem Indikator laden, ist ein Effekt von Entwicklungen, die stark mit Dichtefunktionen zusammenhängen und daher ebenfalls indirekt städtisch-ländlich bzw. zentral-peripher anzeigen.

Auf der Hauptkomponente RC3 laden alle sozialen Indikatoren hoch (Arbeitslosigkeit, der Anteil der Langzeitarbeitslosen und der Anteil der Haushalte mit niedri- gem Einkommen), sodass diese Hauptkomponente die soziale Lage der Bevölkerung in den Städten anzeigt. Das Durchschnittsalter der Bevölkerung ist in den Städten mit hoher sozialer Herausforderung oft infolge selektiver Abwanderungen höher, weshalb die Ladung des Durchschnittsalters auf dieser Hauptkomponente plausibel erscheint. Die positive Ladung des Indikators Abfahrten im öffentlichen Verkehr kann dagegen nur indirekt erklärt werden: Es ist zu vermuten, dass hier auch ein stärkerer Ost-West-Unterschied, sowohl hinsichtlich der sozialen Problemlagen, als auch hinsichtlich der Bedeutung des öffentlichen Verkehrs die Ursache ist.

Hauptkomponente RC4 zeigt das Potenzial der Städte an, welches man marktwirtschaftlich nennen könnte, da sowohl der Arbeitsplatzbesatz und die Pendlerzahlen auf dieser Hauptkomponente positiv laden als auch der Versorgungsgrad der Bevölkerung mit Grunddaseinsvorsorgeeinrichtungen, die von der Privatwirtschaft bereitgestellt werden wie beispielsweise Apotheken und Supermärkte. Ob mehr oder weniger viele Mehrfamilienhäuser das Stadtbild prägen und gebaut werden, ist ebenfalls als Indiz für Dichte und Marktpotenziale zu werten.

Auf die Hauptkomponente RC5 laden nur die beiden kommunalen Steuerindikatoren hoch, die Gewerbesteuern und die Steuerkraft, sodass diese mit wirtschaftlichem Erfolg der Kommune umschrieben werden könnte. Die Hauptkomponente RC6 steht dagegen für die Wohnfunktion, da neben dem Indikator der Wohnfunktion auch das Wanderungssaldo positiv lädt.

Die durchschnittlichen Wegstrecken sowohl der Einals auch der Auspendler, die wir als Attraktivitätsradius und Aktivitätsradius bezeichnen, laden hoch auf die Hauptkomponente RC7. Längere Distanzen sind offensichtlich in den Kommunen anzutreffen, die eine niedrigere Beschäftigtenquote und weniger Abfahrten pro Einwohner per Bahn aufzuweisen haben. Lange Wegstrecken, auch bei den Einpendlern, sind nicht per se mit einer starken Anziehungskraft gleichzusetzen, sondern mit Alltagspraktiken, die in einem eher ländlichen Umfeld mit längeren Wegen verbunden sind. Anders als bei Hauptkomponente RC2 könnte man hier von einer kleinräumigen Zentrum-Peripherie-Achse sprechen.

Die Hauptkomponente RC8 bündelt die Ausstattung mit oberzentralen Einrichtungen wie Hochschulen und Fachhochschulen, allgemeine Bibliotheken und Spezialbibliotheken sowie Shopping-Centern. Die Kinos und Kinositze je Einwohner/-in sind die einzigen Indikatoren, die auf die Hauptkomponente RC9 hochladen und die einzigen Indikatoren für Freizeit- und Kultureinrichtungen. Deshalb scheint es voreilig, diese Hauptkompo- 
Tabelle 2: Ladungen der Indikatoren auf den Hauptkomponenten der PCA auf der Basis von 44 Indikatoren (a) und auf der Basis von 13 Indikatoren (b)

\begin{tabular}{|c|c|c|c|c|c|c|c|c|c|c|}
\hline \multicolumn{11}{|c|}{ Faktorladungen der Indikatoren der globalen Hauptkomponentenanalyse (PCA) } \\
\hline \multirow{2}{*}{$\begin{array}{l}\text { a) vollständiger Datensatz } \\
\text { Indikatoren }\end{array}$} & \multicolumn{10}{|c|}{ Faktoren } \\
\hline & RC1 & RC2 & $\mathrm{RC3}$ & RC4 & RC5 & RC6 & RC7 & RC8 & RC9 & RC10 \\
\hline Jobcenter & 0,680 & 0,053 & 0,148 & 0,126 & $-0,021$ & 0,016 & 0,121 & $-0,024$ & 0,008 & 0,067 \\
\hline Gerichte & 0,680 & 0,009 & 0,075 & 0,046 & $-0,019$ & $-0,017$ & 0,053 & 0,076 & 0,052 & 0,114 \\
\hline berufsbildende Schulen & 0,672 & $-0,087$ & 0,033 & 0,103 & 0,019 & $-0,014$ & 0,058 & 0,241 & 0,085 & 0,105 \\
\hline weiterführende Schulen & 0,594 & $-0,141$ & 0,027 & 0,272 & $-0,029$ & 0,043 & 0,112 & $-0,052$ & $-0,020$ & $-0,010$ \\
\hline Erreichbarkeit von Mittelzentren &,- 0594 & 0,418 & $-0,031$ & 0,197 & 0,010 & $-0,045$ & 0,250 & $-0,166$ & 0,055 & 0,090 \\
\hline Bevölkerungspotenzial & $-0,086$ & $-0,805$ & 0,098 & 0,103 & 0,126 & $-0,039$ & $-0,049$ & 0,024 & $-0,012$ & $-0,045$ \\
\hline Siedlungsdichte & 0,122 & $-0,751$ & 0,036 & 0,353 & 0,049 & $-0,018$ & $-0,229$ & 0,224 & 0,003 & 0,025 \\
\hline Erreichbarkeit von Flughäfen & $-0,020$ & 0,725 & $-0,074$ & 0,161 & $-0,056$ & 0,010 & $-0,007$ & 0,026 & 0,007 & 0,139 \\
\hline Erreichbarkeit von Fernbahnhöfen & $-0,006$ & 0,617 & 0,118 & 0,075 & 0,016 & $-0,075$ & $-0,120$ & $-0,115$ & 0,016 & 0,011 \\
\hline Erreichbarkeit von Autobahnen & $-0,178$ & 0,576 & 0,056 & 0,310 & 0,002 & $-0,087$ & 0,111 &,- 034 & 0,057 & 0,192 \\
\hline Breitbandversorgung & 0,312 & $-0,520$ & $-0,126$ & 0,265 & 0,004 & 0,043 & $-0,026$ &, 055 & 0,010 & 0,025 \\
\hline Erreichbarkeit von Oberzentren & $-0,121$ & 0,491 & 0,247 & 0,159 & $-0,012$ & $-0,097$ & 0,370 &,- 217 & 0,064 & 0,139 \\
\hline Geschlechterproportion & $-0,071$ & $-0,479$ & $-0,080$ & 0,075 & $-0,082$ & 0,174 & $-0,077$ &,- 134 & 0,116 & 0,047 \\
\hline Bibliotheken & $-0,190$ & 0,335 & $-0,233$ & 0,057 & $-0,062$ & $-0,093$ & $-0,082$ & ,135 & 0,071 & $-0,111$ \\
\hline Arbeitslosigkeit & 0,235 & 0,039 & 0,811 & 0,174 & $-0,096$ & $-0,035$ & 0,232 & ,055 & 0,033 & 0,036 \\
\hline Langzeitarbeitslose & 0,112 & $-0,101$ & 0,797 & $-0,034$ & $-0,057$ & $-0,086$ & 0,126 &,- 018 & $-0,037$ & 0,022 \\
\hline Durchschnittsalter & $-0,038$ & 0,379 & 0,674 & 0,137 & $-0,128$ & $-0,011$ & $-0,205$ & $-0,143$ & 0,019 & 0,056 \\
\hline Haushalte mit niedrigem Einkommen & 0,146 & 0,435 & 0,665 & 0,012 & $-0,141$ & $-0,021$ & 0,042 & 0,143 & 0,042 & $-0,007$ \\
\hline Abfahrten im öffentlichen Verkehr & $-0,001$ & $-0,293$ & 0,471 & 0,094 & 0,116 & $-0,105$ & 0,042 & 0,355 & 0,007 & 0,144 \\
\hline Apotheken & 0,288 & 0,021 & 0,119 & 0,642 & $-0,097$ & 0,071 & $-0,015$ & $-0,067$ & 0,086 & $-0,021$ \\
\hline Arbeitsplatzzentralität & 0,476 & 0,036 & 0,072 & 0,553 & 0,295 & 0,014 & 0,201 & 0,199 & 0,139 & 0,054 \\
\hline Supermärkte & 0,008 & 0,409 & 0,067 & 0,547 & $-0,052$ & 0,101 & 0,111 & $-0,120$ & 0,105 & $-0,033$ \\
\hline Innergemeindliche Pendler & 0,520 & 0,064 & 0,235 & 0,521 & 0,097 & 0,022 & 0,310 & 0,317 & 0,082 & 0,038 \\
\hline neu errichtete Mehrfamilienhäuser & 0,119 & $-0,433$ & $-0,109$ & 0,518 & 0,101 & $-0,033$ & 0,028 & 0,212 & 0,128 & 0,102 \\
\hline Pendlersaldo & 0,440 & $-0,070$ & 0,137 & 0,481 & 0,214 & 0,065 & 0,121 & 0,107 & $-0,024$ & $-0,027$ \\
\hline Mehrfamilienhäuser & 0,279 & $-0,355$ & 0,392 & 0,474 & 0,057 & $-0,021$ & $-0,160$ & 0,368 & 0,051 & 0,034 \\
\hline Gewerbesteuer & 0,027 & $-0,027$ & $-0,051$ & 0,088 & 0,934 & $-0,012$ & 0,039 & $-0,007$ & 0,036 & 0,022 \\
\hline Steuerkraft & $-0,008$ & $-0,105$ & $-0,165$ & $-0,027$ & 0,901 & 0,068 & $-0,031$ & $-0,017$ & $-0,006$ & 0,012 \\
\hline Gesamtwanderungssaldo & 0,019 & $-0,090$ & $-0,039$ & 0,073 & 0,029 & 0,891 & $-0,052$ & 0,043 & 0,015 & $-0,033$ \\
\hline Wohnort-Attraktivität & 0,040 & $-0,122$ & $-0,094$ & 0,037 & 0,025 & 0,889 & 0,051 & 0,036 & $-0,012$ & 0,041 \\
\hline Attraktivitätsradius & 0,082 & $-0,024$ & 0,196 & 0,175 & 0,148 & $-0,018$ & 0,742 & 0,152 & 0,115 & $-0,078$ \\
\hline Aktivitätsradius & 0,221 & ,236 & 0,216 & 0,157 & $-0,009$ & 0,076 & 0,732 & 0,133 & 0,102 & $-0,024$ \\
\hline Beschäftigtenquote & $-0,112$ & 0,293 & $-0,006$ & 0,007 & 0,171 & $-0,102$ & $-0,354$ & $-0,118$ & 0,149 & $-0,249$ \\
\hline Bahn-Abfahrten & $-0,059$ & $-0,078$ & 0,142 & 0,085 & 0,043 & 0,057 & $-0,253$ & 0,010 & 0,050 & $-0,080$ \\
\hline Hochschulen & $-0,019$ & $-0,127$ & 0,009 & 0,108 & 0,019 & $-0,001$ & 0,149 & 0,619 & $-0,070$ & 0,060 \\
\hline
\end{tabular}


continued Tabelle 2: Ladungen der Indikatoren auf den Hauptkomponenten der PCA auf der Basis von 44 Indikatoren (a) und auf der Basis von 13 Indikatoren (b)

\begin{tabular}{|c|c|c|c|c|c|c|c|c|c|c|}
\hline \multirow{2}{*}{$\begin{array}{l}\text { a) vollständiger Datensatz } \\
\text { Indikatoren }\end{array}$} & \multicolumn{10}{|c|}{ Faktoren } \\
\hline & RC1 & $\mathrm{RC2}$ & RC3 & RC4 & RC5 & RC6 & RC7 & RC8 & RC9 & RC10 \\
\hline Spezialbibliotheken & 0,062 & 0,045 & 0,010 & 0,005 & $-0,042$ & 0,052 & $-0,021$ & 0,615 & 0,023 & 0,029 \\
\hline Fachhochschulen & 0,139 & $-0,027$ & 0,007 & $-0,022$ & $-0,009$ & 0,009 & 0,041 & 0,470 & 0,086 & 0,000 \\
\hline Shopping Center & 0,189 & $-0,135$ & 0,180 & $-0,017$ & 0,070 & 0,044 & 0,029 & 0,270 & 0,250 & $-0,133$ \\
\hline Kinositzplätze & 0,102 & $-0,023$ & 0,011 & 0,017 & 0,004 & 0,002 & 0,002 & 0,096 & 0,899 & 0,071 \\
\hline Kinos & $-0,012$ & 0,071 & $-0,017$ & 0,217 & 0,020 & $-0,005$ & 0,069 & $-0,005$ & 0,868 & 0,139 \\
\hline Krankenhäuser der Grundversorgung & 0,197 & 0,034 & 0,050 & $-0,040$ & 0,033 & $-0,007$ & 0,056 & $-0,034$ & 0,049 & 0,687 \\
\hline Polizeidienststellen & $-0,085$ & 0,105 & 0,041 & 0,238 & $-0,074$ & $-0,003$ & 0,200 & $-0,151$ & 0,222 & 0,610 \\
\hline Krankenhäuser Spezialversorgung & 0,139 & $-0,014$ & 0,065 & $-0,124$ & 0,044 & 0,025 & $-0,024$ & 0,118 & 0,008 & 0,423 \\
\hline Grundschulen & 0,195 & $-0,122$ & 0,073 & $-0,192$ & 0,001 & 0,036 & 0,233 & $-0,180$ & 0,015 & $-0,420$ \\
\hline b) reduzierter Datensatz & \multicolumn{10}{|c|}{ Faktoren } \\
\hline Indikatoren & RC1 & $\mathrm{RC2}$ & $\mathrm{RC3}$ & RC4 & RC5 & & & & & \\
\hline Mehrfamilienhäuser & 0,862 & 0,262 & 0,012 & 0,067 & $-0,004$ & & & & & \\
\hline Siedlungsdichte & 0,838 & $-0,277$ & 0,040 & $-0,057$ & $-0,086$ & & & & & \\
\hline Pendlersaldo & 0,523 & 0,139 & 0,112 & 0,257 & 0,359 & & & & & \\
\hline Haushalte niedriges Einkommen & $-0,017$ & 0,863 & $-0,067$ & $-0,001$ & 0,068 & & & & & \\
\hline Durchschnittsalter & $-0,092$ & 0,808 & $-0,054$ & ,060 & $-0,070$ & & & & & \\
\hline Arbeitslosigkeit & 0,366 & 0,776 & $-0,059$ & $-0,107$ & 0,169 & & & & & \\
\hline Wanderungssaldo & 0,064 & $-0,040$ & 0,917 & 0,027 & $-0,062$ & & & & & \\
\hline Wohnort-Attraktivität & 0,043 & $-0,108$ & 0,903 & $-0,034$ & 0,100 & & & & & \\
\hline Gewerbesteuer & 0,184 & $-0,254$ & $-0,043$ & 0,615 & 0,078 & & & & & \\
\hline Kinos & 0,018 & 0,115 & 0,042 & 0,589 & 0,176 & & & & & \\
\hline Beschäftigtenquote & $-0,198$ & 0,104 & $-0,056$ & 0,575 & $-0,534$ & & & & & \\
\hline Krankenhäuser der Grundversorgung & $-0,113$ & 0,072 & $-0,043$ & 0,208 & 0,716 & & & & & \\
\hline Schüler an weiterführenden Schulen & 0,439 & 0,040 & 0,086 & 0,037 & 0,476 & & & & & \\
\hline
\end{tabular}

Quelle: Eigene Berechnungen nach INKAR

nente mit Freizeit- und Kulturwert gleichzusetzen, auch wenn keine anderen der verwendeten Indikatoren mit diesen stärker korrelieren.

In der Hauptkomponente RC10 sind schließlich die Versorgung mit Krankenhäusern, sowohl der Grundversorgung als auch die Spezialkliniken, Polizeidienststellen und Grundschulen, vereint. Es handelt sich damit um öffentliche Einrichtungen, die teils in der Fläche, teils in ausgewählten Standorten vorzufinden sind und offensichtlich trotz unterschiedlicher Serviceleistung eine ähnliche räumliche Verteilung aufweisen.
Mit diesen zehn Hauptkomponenten wird die Clusterung der Kleinstädte vorgenommen, deren Ergebnisse im Folgenden beschrieben werden.

\subsection{Ergebnisse der Clusteranalyse}

Die Clusteranalyse als analytischer Schritt zur Gruppierung der Kleinstädte erfolgt nur für die 2.106 Kleinstädte. Auch im Vergleich der Clusterlösungen zwischen den 44 und den 13 Indikatoren ist die Lösung auf der Basis 
des vollständigen Datenmaterials am überzeugendsten, weshalb nur diese als Ergebnis umfassender dargestellt wird.

Zehn von 30 Validitätsindizes schlagen die Bildung von fünf Clustern vor, darunter die Indizes von Caliński und Harabasz (1974) sowie von Duda und Hart (1973). Gemäß fünf Indizes wäre die Bildung von zehn Clustern optimal, je einer empfiehlt sieben, acht oder neun Cluster zu bilden, weitere zwei bewerten sechs Cluster als optimal. Der F-Wert (Verhältnis der mittleren Quadratsumme zwischen den Gruppen zu denen innerhalb der Gruppen) liegt mit 138,2 bei acht Gruppen leicht unter dem von zehn Clustern $(139,1)$ und deutlich unterhalb derer mit fünf Clustern (193,7). Für die Entscheidung für acht statt für zehn Cluster trägt auch dazu bei, dass die zwei zusätzlichen Cluster der 10er-Lösung sich nur wenig von denen unterscheiden, von denen sie gegenüber der 8er-Lösung abgespalten werden.

Werden die Clustermittelpunkte der acht extrahierten Cluster als Startwerte in eine kmeans-Prozedur eingegeben, erhält man eine völlig neue Cluster-Konstellation, die sich wenig mit der Ward-Lösung deckt und zwei extrem schwach besetzte Cluster mit einmal 3 Kleinstädten und einmal 14 Kleinstädten ergibt. Anders dagegen werden bei der Diskriminanzanalyse zwar $20 \%$ der Kleinstädte in benachbarte Cluster neu umsortiert, aber die Diagonale der Kreuztabelle zwischen Ward-Lösung und Diskriminanzprüfung zeigt die hohe Validität der gefundenen Clusterlösung an. Es werden nachfolgend die Cluster nach Korrektur durch die Diskriminanzanalyse weiter betrachtet.

Wie die Mittelwerte und Streuung über die zehn Hauptkomponenten innerhalb und zwischen den Clustern verdeutlichen (vgl. Tabelle 3 ), gibt es einen großen Überschneidungsbereich zwischen allen Kleinstädten. Die Cluster unterscheiden sich vornehmlich dadurch, dass ein bis zwei Hauptkomponenten pro Cluster überdurchschnittliche Mittelwerte aufweisen. Da auch hier, ähnlich wie bei der Hauptkomponentenbezeichnung, die Benennung schwierig ist, werden die Cluster mit den Buchstaben $\mathrm{A}$ bis $\mathrm{H}$ gekennzeichnet. Allein die Hauptkomponente RC4 trägt nicht zur Unterscheidung der Kleinstadttypen bei.

Cluster A zeichnet sich beispielsweise durch überdurchschnittliche Werte der Hauptkomponente RC5 (wirtschaftlicher Erfolg; Mittelwert $=4,975$ ) aus. Es handelt sich um eine kleine Gruppe von 30 Kleinstädten. Leicht überdurchschnittlich ist der Mittelwert in der Hauptkomponente RC3 (soziale Lage) im Cluster B $(0,834)$, weit überdurchschnittlich im Cluster $H(1,452)$. Eine herausragende Stellung oberzentraler Einrichtun- gen (Hauptkomponente RC8) findet sich bei den Kleinstädten des Clusters $G$ (Mittelwert $=3,966$ ). Dieses Cluster ist ebenfalls eine kleine Gruppe von insgesamt 40 Kleinstädten.

Cluster E ist das zahlenmäßig größte Cluster mit 997 Kleinstädten. Die Hauptkomponente RC2 (ZentrumPeripherie) weist im Mittel in diesem Cluster die niedrigsten Werte auf $(-0,622)$. Die Pkw-Fahrzeiten zu oberzentralen Einrichtungen sind am geringsten; es handelt sich um eine Gruppe eher zentral gelegener Kleinstädte. Cluster C mit 201 Kleinstädten weist eine gewisse Spezifik bei den mittelzentralen (Hauptkomponente RC1; Mittelwert $=2,458$ ) und öffentlichen Einrichtungen (Hauptkomponente RC10; Mittelwert $=0,774$ ) auf. Cluster D fällt durch eine überdurchschnittliche Wohnfunktion und ein hohes Wanderungssaldo (Hauptkomponente RC6; Mittelwert $=0,911$ ) bei gleichzeitig unterdurchschnittlichen Werten in der sozialen Lage (Hauptkomponente RC3; Mittelwert $=-0,729$ ) auf.

Abbildung 4 verortet die Kleinstädte gemäß ihrer Clusterzugehörigkeit in der Bundesrepublik. Das Kartenbild zeigt eine plausible Verteilung der gefundenen Cluster. So gruppieren sich die Kleinstädte des Clusters E in der Tat im Umkreis der Großstädte und innerhalb der Agglomerationen.

Auffällig ist die Konzentration des Kleinstadttyps $\mathrm{H}$ auf die südliche Hälfte und des Kleinstadttyps B auf die nördliche Hälfte Ostdeutschlands. Dabei sind beide Cluster keine reinen ostdeutschen Typen, sondern auch in Westdeutschland anzutreffen. Die Cluster B, D und $\mathrm{H}$ unterscheiden sich vornehmlich durch den Grad, wie stark sie durch Bevölkerung in prekärer, sozialer Lage betroffen sind bzw. durch Anteile an Bevölkerungsschichten der mittleren und höheren Einkommensgruppen. Sie verteilen sich daher relativ konzentriert im Raum, wobei es jedoch auch fließende Übergänge zwischen den Kleinstadttypen gibt. Die Kleinstädte mit sehr spezifischen Ausstattungen (erfolgreiche Wirtschaftsunternehmen (A), Mittelzentrale und öffentliche Einrichtungen $(C)$, oberzentrale Einrichtungen $(G)$ ) verteilen sich über das gesamte Bundesgebiet. Für eine herausragende Stellung einer Kleinstadt ist daher inr infrastrukturelles Kapital bedeutsam, worunter vor allem zentralörtliche Einrichtungen und Wirtschaftsunternehmen zu nennen sind. Mit der Stärkung der zentralen Orte durch Sicherung der Einrichtungen zur Daseinsvorsorge sichert man die Funktionalität der Kleinstädte und des Umlandes, schafft jedoch noch keine Alleinstellungsmerkmale, die auch für die Zukunftsfähigkeit der Städte und ihre oft zitierten Ankerfunktionen der Regionalentwicklung relevant sind. In Übereinstimmung mit Maillat (1998: 125- 
Tabelle 3: Mittelwerte und Standardabweichungen der Hauptkomponenten RC1 bis RC10 für die Lösung mit acht Clustern

\begin{tabular}{|c|c|c|c|c|c|c|c|c|c|c|c|}
\hline \multirow{2}{*}{ Cluster } & & \multicolumn{10}{|c|}{ Durchschnittliche Scores der zehn Hauptkomponenten (PCA) } \\
\hline & & RC1 & RC2 & RC3 & RC4 & RC5 & RC6 & RC7 & RC8 & RC9 & RC10 \\
\hline \multirow{2}{*}{$\begin{array}{l}\text { A } \\
(30)\end{array}$} & Mittelwert & $-0,186$ & $-0,499$ & $-0,303$ & 0,250 & 4,975 & 0,162 & $-0,001$ & $-0,335$ & $-0,176$ & $-0,116$ \\
\hline & Standardabweichung & 0,881 & 0,963 & 0,989 & 1,179 & 5,127 & 0,912 & 0,747 & 0,570 & 0,515 & 0,742 \\
\hline \multirow{2}{*}{$\begin{array}{l}\text { B } \\
(238)\end{array}$} & Mittelwert & $-0,171$ & 0,614 & 0,834 & 0,446 & $-0,219$ & 0,324 & 1,361 & $-0,383$ & $-0,321$ & 0,153 \\
\hline & Standardabweichung & 0,838 & 0,680 & 0,817 & 1,160 & 0,412 & 0,797 & 1,072 & 0,442 & 0,364 & 0,856 \\
\hline \multirow{2}{*}{$\begin{array}{l}\text { C } \\
(201)\end{array}$} & Mittelwert & 2,458 & 0,428 & $-0,207$ & 0,184 & $-0,154$ & 0,176 & 0,005 & $-0,497$ & 0,011 & 0,774 \\
\hline & Standardabweichung & 1,110 & 0,692 & 0,874 & 0,814 & 0,496 & 0,727 & 0,839 & 0,681 & 0,680 & 1,309 \\
\hline \multirow{2}{*}{$\begin{array}{l}D \\
(314)\end{array}$} & Mittelwert & 0,034 & 0,193 & $-0,729$ & 0,061 & $-0,033$ & 0,911 & 0,226 & $-0,196$ & $-0,204$ & $-0,433$ \\
\hline & Standardabweichung & 0,539 & 0,597 & 0,641 & 0,645 & 0,503 & 0,884 & 0,832 & 0,396 & 0,259 & 0,494 \\
\hline \multirow{2}{*}{$\begin{array}{l}\text { E } \\
(997)\end{array}$} & Mittelwert & $-0,329$ & $-0,622$ & $-0,219$ & 0,116 & $-0,128$ & $-0,185$ & $-0,140$ & $-0,272$ & $-0,197$ & 0,046 \\
\hline & Standardabweichung & 0,572 & 0,708 & 0,570 & 0,680 & 0,451 & 0,681 & 0,758 & 0,399 & 0,272 & 0,602 \\
\hline \multirow{2}{*}{$\begin{array}{l}\mathrm{F} \\
(78)\end{array}$} & Mittelwert & $-0,019$ & $-0,182$ & $-0,094$ & $-0,068$ & 0,065 & 0,411 & $-0,041$ & $-0,074$ & 2,202 & $-0,158$ \\
\hline & Standardabweichung & 0,801 & 0,788 & 0,808 & 1,139 & 0,992 & 0,817 & 0,774 & 0,695 & 1,995 & 1,158 \\
\hline & Mittelwert & $-0,091$ & 0,505 & $-0,328$ & $-0,230$ & $-0,195$ & 0,431 & $-0,199$ & 3,966 & $-0,031$ & 0,257 \\
\hline & Standardabweichung & 1,396 & 1,137 & 0,800 & 0,983 & 0,825 & 0,685 & 1,148 & 2,529 & 1,775 & 0,900 \\
\hline \multirow{2}{*}{$\begin{array}{l}\mathrm{H} \\
(208)\end{array}$} & Mittelwert & $-0,100$ & 0,642 & 1,452 & 0,150 & 0,038 & $-0,294$ & $-0,834$ & $-0,168$ & $-0,131$ & $-0,484$ \\
\hline & Standardabweichung & 0,650 & 0,560 & 0,541 & 0,822 & 0,409 & 0,900 & 0,959 & 0,345 & 0,319 & 0,517 \\
\hline \multirow{2}{*}{$\begin{array}{l}\text { Kleinstädte } \\
(2.106)\end{array}$} & Mittelwert & 0,049 & $-0,097$ & $-0,008$ & 0,143 & $-0,032$ & 0,098 & 0,034 & $-0,198$ & $-0,093$ & $-0,002$ \\
\hline & Standardabweichung & 1,066 & 0,876 & 0,915 & 0,814 & 0,986 & 0,863 & 1,005 & 0,814 & 0,729 & 0,816 \\
\hline
\end{tabular}

Quelle: Eigene Berechnungen nach INKAR

126) benötigen städtische Systeme vier herausragende Eigenschaften: institutionelle Funktionen, städtische Baukultur, städtische Symbolfunktionen und Produktionsfunktionen zur Entfaltung ihres vollen endogenen Entwicklungspotenzials. Kleinstädte verfügen nur zum Teil über alle städtischen Funktionen. Die Kleinstadttypen zeigen, in welchen Kleinstädten diese in Ansätzen und wo in herausragendem Maße gegeben sind. Die hier herausgearbeiteten Kleinstadttypen bedürfen jedoch weiterer Analysen, um zielführendere Anleitungen für die Stadtentwicklungspolitik geben zu können.

\section{Diskussion und Forschungsbedarf}

Für diese Studie wurden für alle Einheitsgemeinden und Gemeindeverbände 44 Indikatoren ausgewählt, die zu zehn Hauptkomponenten gebündelt wurden. Mit diesen Hauptkomponenten konnten die 2.106 Kleinstädte in
Deutschland über eine Clusteranalyse in acht Kleinstadttypen aufgeteilt und mit anschließender Diskriminanzanalyse validiert werden.

Die Auswahl der Indikatoren und/oder Komponenten ist für die Kleinstadttypisierung der erste elementare Schritt. Hier wurde ein möglichst breiter Katalog gewählt, der alle in der Literatur als relevant erachteten Kriterien einschließt. Herausgekommen sind Cluster, die jeweils in ein oder zwei der extrahierten Hauptkomponenten deutlich vom Mittelwert abweichende Werte aufweisen. Nur die Hauptkomponente, die hier als marktwirtschaftliches Potenzial umschrieben wird, hilft nicht zur Unterscheidung zwischen den Kleinstädten. Man kann dieses Ergebnis dahingehend interpretieren, dass eine gewisse Arbeitsplatzzentralität, ein gewisses Aufkommen an Einpendlern sowie die Bereitstellung von Grunddienstleistungen zur Charakteristik einer Kleinstadt allgemein bedeutsam ist, aber darüber hinaus keine weitere funktionale Unterscheidung zwischen Kleinstädten ermöglicht. 


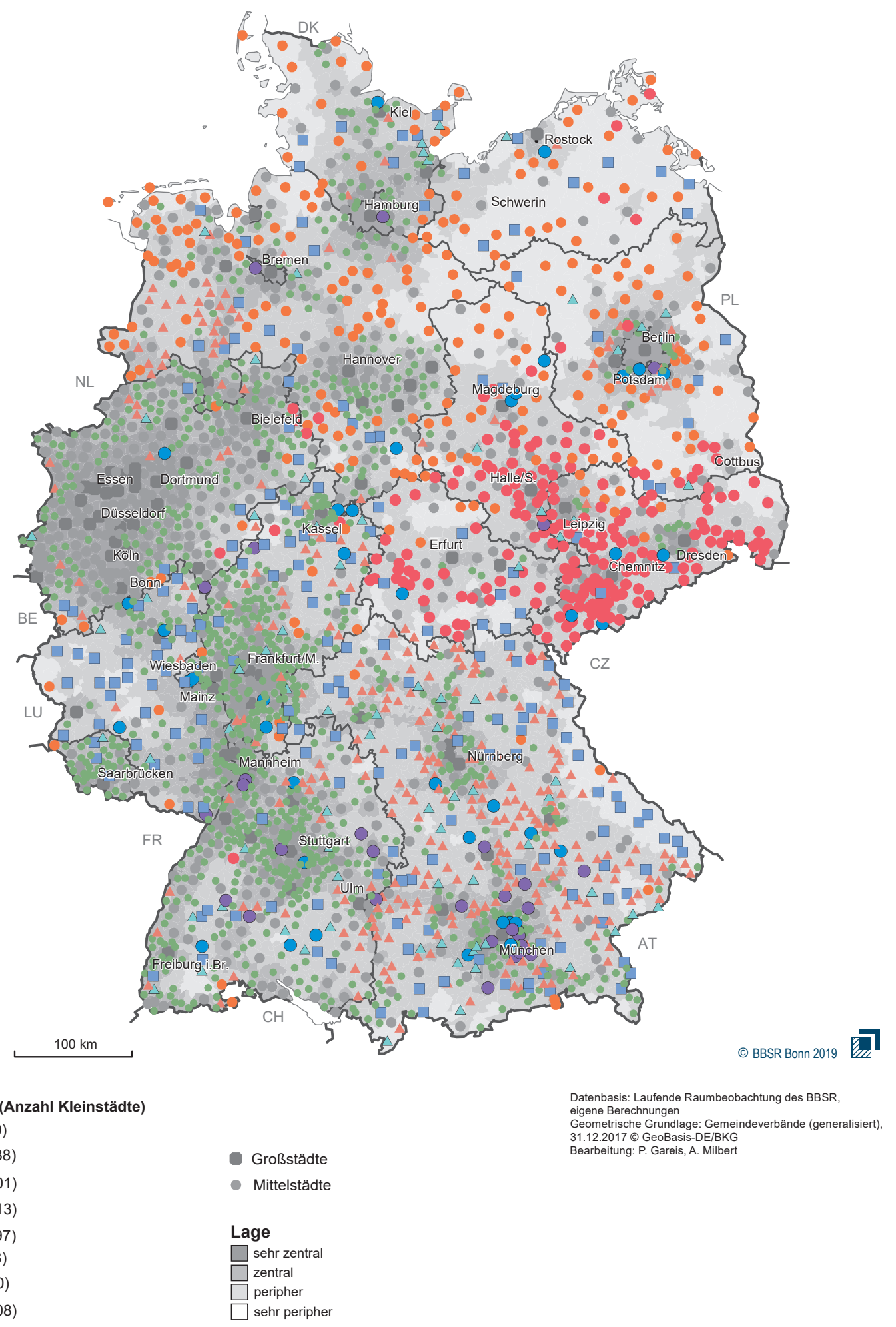

Abbildung 4: Kleinstadttypen in Deutschland

Quelle: Eigene Berechnungen nach INKAR; Kartengrundlage: Bundesamt für Kartographie und Geodäsie ${ }^{7}$

7 https://gdz.bkg.bund.de/index.php/default/digitale-geodaten/verwaltungsgebiete/verwaltungsgebiete-1-25-000-ebenen-stand-31-12vg25-ebenen.html (12.09.2020). 
Bislang noch nicht hinreichend gelöst ist die Einbeziehung des räumlichen Kontextes, in dem sich die Kleinstädte befinden. Daher wird in diesem Beitrag mithilfe der globalen Hauptkomponentenanalyse (PCA) sowie der geographisch gewichtete Hauptkomponentenanalysen (GWPCA) versucht, eine Einordnung der Kleinstädte in ihrer funktionalen Ausstattung und ihrem räumlichen Kontext zu untersuchen. Die Anwendung der GWPCA stößt auch mit dem reduzierten Indikatorenkatalog mit 13 Variablen hinsichtlich der Interpretierbarkeit der Ergebnisse im Vergleich zur PCA an ihre Grenzen. Es ergeben sich zwar teilweise relativ ähnliche Indikatorenkonstellationen hinsichtlich der Hauptkomponentenladungen, die mit der PCA vergleichbar sind. Jedoch weisen die Hauptkomponentenladungen grundsätzlich geringere Werte auch bei den höchst ladenden Indikatoren auf, die nicht selten unter 0,4 liegen und größtenteils deutlich diffuser (auf mehrere Hauptkomponenten verteilt) sind als bei Einsatz der PCA. Hier zeigt sich, dass der Einbezug der umliegenden Gemeinden zu viele Aspekte in der Analyse miteinzubeziehen versucht. Im Vergleich zu den bisherigen Forschungsvorhaben, in denen die GWPCA zum Einsatz kam (vgl. Lloyd 2010: 391; Li/Cheng/Wu 2016: 236-237; Wei/Cabrera-Barona/ Blaschke 2016: 981; Tsutsumida/Harris/Comber 2017: 1062; Losada/Alen/Cotos-Yáñez et al. 2019: 446; Wu/ Hu/Zhou et al. 2019: 175), ist diese Untersuchung mit Abstand am umfangreichsten sowohl hinsichtlich der Anzahl räumlicher Untersuchungseinheiten als auch hinsichtlich der Anzahl an gewählten Indikatoren. In dieser Kombination ist das Verfahren (noch) kaum zu steuern.

Es ist weitere Erfahrung im Umgang mit der GWPCA notwendig, um eine höhere Validität mittels neuer Testverfahren zu erlangen und auch die Interpretationsfähigkeit der Ergebnisse zu verbessern. Monte-CarloAnsätze zur Bestimmung der Güte der GWPCA konnten gar nicht erst berechnet werden, da die Distanzmatrizen zu groß waren. Neuere Ansätze zur Validitätsprüfung liegen bereits vor (Roca-Pardiñas/Ordóñez/CotosYáñez et al. 2017: 681-682), wurden jedoch noch nicht in Softwarelösungen implementiert. Elementar ist dabei weiterhin die Auswahl und Bestimmung der Bandbreite, welche in zukünftigen Analysen besondere Beachtung finden muss. Auch das Ausgangsmaterial an Indikatoren muss sehr sorgfältig ausgewählt werden. Daher ist die GWPCA nicht prinzipiell für derartige Analysen zu verwerfen, sie bedarf jedoch weiterer empirischer Praxis, um anwendungs- und fragestellungsbezogen eingesetzt werden zu können.

Die klassischen Verfahren, wie sie in diesem Fall bevorzugt wurden - PCA und Clusteranalyse, ergänzt um die Diskriminanzanalyse zur Validierung der Clusterlösung - führen zu plausiblen Ergebnissen. Es konnten signifikant unterschiedliche Cluster bzw. Kleinstadttypen gefunden werden. Somit konnten Cluster identifiziert werden, die jenseits der pauschalen Logik der Lage „zentral versus peripher" weitere Faktoren bestimmen, die für die Stellung und Funktionen von Kleinstädten relevant sind, unter anderem eine Ausstattung mit mittelzentralen oder teils oberzentralen Einrichtungen, starke Unternehmen sowie die soziale Lage der Kleinstädte. Viele Kleinstädte stellen zwar Mischformen aus mehreren der betrachteten Hauptkomponenten dar, dennoch scheint es unter den Kleinstädten dominierende Faktoren zu geben, die diese charakterisieren.

Prinzipiell bestätigen die Ergebnisse, dass auch die multivariaten, auf quantitativen Daten gestützten Verfahren hilfreich sind, Erfolgsfaktoren für die Kleinstadtentwicklung zu extrahieren. Insbesondere aber zeigen die Ergebnisse, dass Kleinstädte auch objektiv gesehen sehr unterschiedliche Ausgangsvoraussetzungen haben. Die bisher stärker fallstudienbezogene Kleinstadtforschung sollte sich den quantitativen Methoden gegenüber offener zeigen. Da die quantitativen Verfahren in der Großstadtforschung einerseits sowie in der Regionalanalyse andererseits zwar erprobt sind, nicht jedoch ausreichend in der Kleinstadtforschung Anwendung finden, kristallisiert sich diesbezüglich folgender weiterer Forschungsbedarf heraus:

- Die hier und in der Literatur verwendeten Kennzahlen sind immer noch stark bedingt durch das vorliegende Datenangebot. Neuere Daten, die z. B. auch die innerstädtischen Differenzierungen und Segregationen in Kleinstädten berücksichtigen, konnten wegen fehlender Daten noch nicht einbezogen werden. Dabei könnten insbesondere OpenStreetMap-Daten hilfreich sein, deren Validität sich in den vergangenen Jahren deutlich verbessert haben und einen umfangreichen Funktionskatalog zur Analyse von (Klein-)Städten bereitstellen (Zhang/ Pfoser 2019). Hier besteht erhöhter Forschungs- und Datenbedarf.

- Die gewählten Verfahren sind grundsätzlich hilfreich, Kleinstadttypen zu bestimmen. Allerdings unterliegen die gefundenen Typen immer auch einer gewissen Spannbreite. Je höher die Anzahl der Untersuchungseinheiten und Kriterien, desto wahrscheinlicher sind Überlappungen in den Typen. Von Typen wird jedoch erwartet, dass sie eindeutig sind. Eine Schärfung der Kriterienauswahl wäre der erste Schritt zu einer stärkeren Profilierung der Kleinstadttypen. Für die weitere Forschung können 
die hier vorgelegten Ergebnisse erste Anhaltspunkte bezüglich der einzubeziehenden Funktionen liefern.

- Der Beitrag zeigt, dass der Versuch, erstmals die GWPCA anzuwenden, fehlschlug: zum einen aufgrund der sehr schwierig zu interpretierenden Ergebnisse und zum anderen wegen der berechneten, sehr hohen Bandbreiten. Hier gilt es, die GWPCA im Kontext von funktionell sehr umfangreichen Klassifikationen in weiteren Forschungsvorhaben zu schärfen und erneut zu erproben sowie im Hinblick auf ihren methodischen Einsatz zu bewerten. Insbesondere weitere regionale Untersuchungen könnten hier die Anwendbarkeit der Methode schärfen.

\section{Literatur}

Adam, B.; Berndgen-Kaiser, A.; Jochimsen, K.; Münter, A.; Zakrzewski,P.(2018): ÄltereEin-undZweifamilienhausbestände im Umbruch. Eine Clusteranalyse zur Identifizierung regionaler Betroffenheiten in Nordrhein-Westfalen. In: Raumforschung und Raumordnung | Spatial Research and Planning 76, 1, 3-17. doi: 10.1007/s13147-017-0479-y

Andrews, H. F. (1971): A cluster analysis of British towns. In: Urban Studies 8, 3, 271-284. doi: 10.1080/00420987120080451

Aring, J. (2003): Stadt-Umland-Wanderung und die Ausdifferenzierung der Wohnungsmärkte. In: vhw Forum Wohneigentum 5, 268-275.

Backhaus, K.; Erichson, B.; Plinke, W.; Weiber, R. (2016): Multivariate Analysemethoden. Berlin. doi: 10.1007/978-3-66246076-4.

Bähr, J. (1971): Gemeindetypisierung mit Hilfe quantitativer statistischer Verfahren (Beispiel: Regierungsbezirk Köln). In: Erdkunde 25, 4, 249-264. doi: 10.3112/erdkunde.1971.04.02

BBSR - Bundesinstitut für Bau-, Stadt- und Raumforschung (2016): Regionale Disparitäten in Deutschland auf der Ebene von Gemeinden und Kreisen: Expertise zum Siebten Altenbericht der Bundesregierung. Berlin.

Bell, D.; Jayne, M. (2009): Small cities? Towards a research agenda. In: International Journal of Urban and Regional Research 33, 3, 683-699. doi: 0.1111/j.1468-2427.2009.00886.x

Blotevogel, H. H. (2006): Gemeindetypisierung NordrheinWestfalens nach demographischen Merkmalen. In: Danielzyk, R.; Kilper, H. (Hrsg.): Demographischer Wandel in ausgewählten Regionstypen Nordrhein-Westfalens Herausforderungen und Chancen für regionale Politik. Hannover, 17-33. = Arbeitsmaterial der ARL 329.

Brombach, K.; Jessen, J. (2005): Kleinstädte in Großstadtregionen. Funktionsräumliche Spezialisierung und Zentrumsfunktion am Beispiel Region Stuttgart. In: Informationen zur Raumentwicklung 8, 477-490.

Caliński, T.; Harabasz, J. (1974): A dendrite method for cluster analysis. In: Communications in Statistics 3, 1, 1-27.

Charrad, M.; Ghazzali, N.; Boiteau, V.; Niknafs, A. (2014): NbClust: An $R$ Package for Determining the Relevant Number of
Clusters in a Data Set. In: Journal of Statistical Software 61, 6, 1-36. doi: 10.18637/jss.v061.i06

Demšar, U.; Harris, P.; Brunsdon, C.; Fotheringham, A. S.; McLoone, S. (2013): Principal Component Analysis on Spatial Data: An Overview. In: Annals of the Association of American Geographers 103, 1, 106-128. doi: 10.1080/00045608.2012.689236

Duda, R. O.; Hart, P. E. (1973): Pattern Classification and Scene Analysis. New York.

Empirica (2017): Wohnen und Baukultur nicht nur in Metropolen. Hintergrundpapier zum gleichnamigen Positionspapier der Bundesstiftung Baukultur und dem GdW Bundesverband deutscher Wohnungs- und Immobilienunternehmen. Berlin.

Fehlberg, T.; Mießner, M. (2015): Mietpreissteigerungen und Wohnungsengpässe abseits der Ballungsräume. Investitionen in Wohnimmobilien in B-Lagen und Regionalzentren - das Beispiel Göttingen. In: sublurban. Zeitschrift für kritische Stadtforschung 3, 1, 25-44.

Fotheringham, A. S.; Brunsdon, C.; Charlton, M. (2002): Geographically weighted regression: The analysis of spatially varying relationships. Chichester.

Gatzweiler, H.-P.; Adam, B.; Milbert, A.; Pütz, T.; Spangenberg, M.; Sturm, G.; Walther, A. (2012): Klein- und Mittelstädte in Deutschland - eine Bestandsaufnahme. Bonn. = Analysen Bau.Stadt.Raum 10.

Geyler, S.; Warner, B.; Brandl, A.; Kuntze, M. (2008): Clusteranalyse der Gemeinden in der Kernregion Mitteldeutschland. Eine Typisierung der Region nach Entwicklungsparametern und Rahmenbedingungen. Leipzig. = Schriftenreihe des Forschungsverbundes KoReMi 2.

Göddecke-Stellmann, J. (2011): Renaissance der Großstädte eine Zwischenbilanz. Bonn. = BBSR-Berichte Kompakt 9/2011.

Gollini, I.; Lu, B.; Charlton, M.; Brunsdon, C.; Harris, P. (2015): GWmodel: An R Package for Exploring Spatial Heterogeneity Using Geographically Weighted Models. In: Journal of Statistical Software 63, 17, 1-50. doi: 10.18637/jss.v063.i17

Grove, D. M.; Roberts, C. A. (1980): Principal component and cluster analysis of 185 large towns in England and Wales. In: Urban Studies 17, 1, 77-82. doi: 10.1080/00420988020080091

Harfst, J.; Wirth, P. (2014): Zur Bedeutung endogener Potenziale in klein- und mittelstädtisch geprägten Regionen - Überlegungen vor dem Hintergrund der Territorialen Agenda 2020. In: Raumforschung und Raumordnung 72, 6, 463-475. doi: 10.1007/s13147-014-0312-9

Harris, C. D. (1943): A functional classification of cities in the United States. In: Geographical Review 33, 1, 86-99. doi: $10.2307 / 210620$

Harris, P.; Brunsdon, C.; Charlton, M. (2011): Geographically weighted principal components analysis. In: International Journal of Geographical Information Science 25, 10, 1717-1736. doi: 10.1080/13658816.2011.554838

Harris, P.; Clarke, A.; Juggins, S.; Brunsdon, C.; Charlton, M. (2015): Enhancements to a geographically weighted principal component analysis in the context of an application to an environmental data set. In: Geographical Analysis 47, 2, 146172. doi: 10.1111/gean.12048

Herrenknecht, A.; Wohlfahrt, J. (2005): Die Bibliographie zur ländlichen Kleinstadt 1945-2005. In: Pro-Regio-Online: Zeitschrift für den ländlichen Raum 3, 5-14. 
Jiang, S.; Alves, A.; Rodrigues, F.; Ferreira Jr., J.; Pereira, F. C. (2015): Mining point-of-interest data from social networks for urban land use classification and disaggregation. In: Computers, Environment and Urban Systems 53, 36-46. doi: 10.1016/j.compenvurbsys.2014.12.001

Kühn, M.; Milstrey, U. (2015): Mittelstädte als periphere Zentren: Kooperation, Konkurrenz und Hierarchie in schrumpfenden Regionen. In: Raumforschung und Raumordnung 73, 3, 185202. doi: $10.1007 / \mathrm{s} 13147-015-0343-x$

Leibert, T. (2019): Demographische Strukturen und Entwicklungen. In: Porsche, L.; Steinführer, A.; Sondermann, M. (Hrsg.): Kleinstadtforschung in Deutschland: Stand, Perspektiven und Empfehlungen. Hannover, 20-22. = Arbeitsberichte der ARL 28.

Li, Z.; Cheng, J.; Wu, Q. (2016): Analyzing regional economic development patterns in a fast developing province of China through geographically weighted principal component analysis. In: Letters in Spatial and Resource Sciences 9, 3, 233-245. doi: 10.1007/s12076-015-0154-2

Lloyd, C. D. (2010): Analysing population characteristics using geographically weighted principal components analysis: a case study of Northern Ireland in 2001. In: Computers, Environment and Urban Systems 34, 5, 389-399. doi: 10.1016/j. compenvurbsys.2010.02.005

Losada, N.; Alen, E.; Cotos-Yáñez, T. R; Domínguez, T. (2019): Spatial heterogeneity in Spain for senior travel behavior. In: Tourism Management 70, 444-452. doi: 10.1016/j. tourman.2018.09.011

Maillat, D. (1998): Interactions between urban systems and localized productive systems: An approach to endogenous regional development in terms of innovative milieu. In: European Planning Studies 6, 2, 117-129. doi: 10.1080/09654319808720450

Meili, R.; Mayer, H. (2017): Small and medium-sized towns in Switzerland. Economic heterogeneity, socioeconomic performance and linkages. In: Erdkunde 71, 4, 313-332. doi: 10.3112/erdkunde.2017.04.04.

Messerli, P. (1999): Sind die Alpenstädte besondere Städte? In: Revue de Géographie Alpine 87, 2, 65-76.

Moser, C. A.; Scott, W. (1961): British towns: a statistical study of their social and economic differences. Edinburgh.

Porsche, L.; Milbert, A. (2018): Kleinstädte in Deutschland. Ein Überblick. In: Informationen zur Raumentwicklung 6, 4-21.

Porsche, L.; Milbert, A.; Steinführer, A. (2019): Einführung. In: Porsche, L.; Steinführer, A.; Sondermann, M. (Hrsg.): Kleinstadtforschung in Deutschland: Stand, Perspektiven und Empfehlungen. Hannover, 5-15. = Arbeitsberichte der ARL 28.

Porsche, L.; Steinführer, A.; Dehne, P.; Sondermann, M. (2019): Schlussfolgerungen und zentrale Empfehlungen. In: Porsche, L.; Steinführer, A.; Sondermann, M. (Hrsg.): Kleinstadtforschung in Deutschland: Stand, Perspektiven und Empfehlungen. Hannover, 52-56. = Arbeitsberichte der ARL 28.

Reichert-Schick, A. (2010): Auswirkungen des demographischen Wandels in regionaler Differenzierung: Gemeinsamkeiten und Gegensätze ländlich-peripherer Entleerungsregionen in Deutschland - die Beispiele Vorpommern und Westeifel. In: Raumforschung und Raumordnung 68, 3, 153-168. doi: 10.1007/s13147-010-0027-5
Roca-Pardiñas, J.; Ordóñez, C.; Cotos-Yáñez; T. R.; Pérez-Álvarez, R. (2017): Testing spatial heterogeneity in geographically weighted principal components analysis. In: International Journal of Geographical Information Science 31, 4, 676-693. doi: 10.1080/13658816.2016.1224886

Schmitz-Veltin, A. (2015): Reurbanisierung im Kontext einer neuen Unübersichtlichkeit regionaler Entwicklungsmuster - Das Beispiel der Stadtregion Stuttgart. In: Fricke, A.; Siedentop, S.; Zakrzewski, P. (Hrsg.): Reurbanisierung in baden-württembergischen Stadtregionen. Hannover, 77-95. =Arbeitsberichte der ARL 14.

Schneider, W.; Scheibler, D. (1983): Probleme und Möglichkeiten bei der Bewertung von Clusteranalyse-Verfahren. I. Ein Überblick über einschlägige Evaluationsstudien. In: Psychologische Beiträge 25, 1-2, 208-237.

Schultz, A.; Brandt, P. (2017): Demographietypisierung. Methodisches Vorgehen und empirische Befunde. https://www. wegweiser-kommune.de/documents/10184/10615/Methodik Clusterung.pdf/05a1b137-7dbf-4bf4-828d-9a097a4f3805 (06.09.2020).

Schulze, M.; Margraf, O. (2000): Eine sozio-ökonomische Typisierung der russischen Städte während der Transformationsphase. In: Europa Regional 8, 1, 43-61.

Schwippe, H. J. (1979): Faktorenanalyse und Clusteranalyse. Möglichkeiten des Einsatzes multivariater Verfahren in der Analyse des Verhältnisses von Stadt und Land im östlichen Münsterland im frühen 19. Jahrhundert. In: Schröder, W. H. (Hrsg.): Moderne Stadtgeschichte. Stuttgart, 112-144.

Servillo, L.; Atkinson, R.; Smith, I.; Russo, A.; Sýkora, L.; Demazière, C.; Hamdouch A. (2014): TOWN. Small and medium sized towns in their functional territorial context. Final Report. Luxemburg.

Smith, R. H. T. (1965): Method and purpose in functional town classification. In: Annals of the Association of American Geographers 55, 3, 539-548.

Steinführer, A.; Porsche, L.; Sondermann, M. (2019): Anlass und Zielstellung. In: Porsche, L.; Steinführer, A.; Sondermann, M. (Hrsg.): Kleinstadtforschung in Deutschland: Stand, Perspektiven und Empfehlungen. Hannover, 3-4. = Arbeitsberichte der ARL 28.

Tsutsumida, N.; Harris, P.; Comber, A. (2017): The Application of a Geographically Weighted Principal Component Analysis for Exploring Twenty-three Years of Goat Population Change across Mongolia. In: Annals of the American Association of Geographers 107, 5, 1060-1074. doi: 10.1080/24694452.2017.1309968

Volgmann, K. (2014): Entwicklung metropolitaner Funktionen im polyzentralen deutschen Städtesystem - Raummuster der Konzentration und funktionalen Spezialisierung. In: Raumforschung und Raumordnung 72, 1, 21-37. doi: 10.1007/ s13147-013-0264-5

Volgmann, K.; Rusche, K. (2020): The Geography of Borrowing Size: Exploring Spatial Distributions for German Urban Regions. In: Tijdschrift voor Economische en Sociale Geografie 111, 1, 60-79. doi:10.1111/tesg.12362

Wei, C.; Cabrera-Barona, P.; Blaschke, T. (2016): Local geographic variation of public services inequality: does the neighborhood scale matter? In: International Journal of Environmental Research and Public Health 13, 10, 981. doi: 10.3390/ ijerph13100981 
Winkler-Kühlken, B.; Diller, C.; Gareis, P.; Lenk, J.; von Popowski, M. (2019): Lage und Zukunft der Kleinstädte in Deutschland Bestandsaufnahme zur Situation der Kleinstädte in zentralen Lagen. Bonn. = BBSR-Online-Publikation 15/2019.

Wirth, P.; Elis, V.; Müller, B.; Yamamoto, K. (2016): Peripheralisation of small towns in Germany and Japan - Dealing with economic decline and population loss. In: Journal of Rural Studies 47, 62-75. doi: 10.1016/j.jrurstud.2016.07.021

Wolff, H.-G.; Bacher, J. (2010): Hauptkomponentenanalyse und explorative Faktorenanalyse. In: Wolf, C.; Best, H. (Hrsg.): Handbuch der sozialwissenschaftlichen Datenanalyse. Wiesbaden, 333-365. doi: 10.1007/978-3-531-92038-2_15

Wu, C.; Hu, W.; Zhou, M.; Li, S.; Jia, Y. (2019): Data-driven regionalization for analyzing the spatiotemporal characteristics of air quality in China. In: Atmospheric Environment 203, 172182. doi: 10.1016/j.atmosenv.2019.01.048

Zhang, L.; Pfoser, D. (2019): Using OpenStreetMap point-of-interest data to model urban change - A feasibility study. In: PloS one 14, 2. doi: 10.1371/journal.pone.0212606 